\title{
BAYESIAN INFERENCE VIA CLASSES OF NORMALIZED RANDOM MEASURES
}

\author{
Lancelot F. James, Antonio Lijoi and Igor Prünster ${ }^{1}$ \\ Hong Kong University of Science and Technology and University of Pavia
}

\begin{abstract}
One of the main research areas in Bayesian Nonparametrics is the proposal and study of priors which generalize the Dirichlet process. Here we exploit theoretical properties of Poisson random measures in order to provide a comprehensive Bayesian analysis of random probabilities which are obtained by an appropriate normalization. Specifically we achieve explicit and tractable forms of the posterior and the marginal distributions, including an explicit and easily used description of generalizations of the important Blackwell-MacQueen Pólya urn distribution. Such simplifications are achieved by the use of a latent variable which admits quite interesting interpretations which allow to gain a better understanding of the behaviour of these random probability measures. It is noteworthy that these models are generalizations of models considered by Kingman (1975) in a non-Bayesian context. Such models are known to play a significant role in a variety of applications including genetics, physics, and work involving random mappings and assemblies. Hence our analysis is of utility in those contexts as well. We also show how our results may be applied to Bayesian mixture models and describe computational schemes which are generalizations of known efficient methods for the case of the Dirichlet process. We illustrate new examples of processes which can play the role of priors for Bayesian nonparametric inference and finally point out some interesting connections with the theory of generalized gamma convolutions initiated by Thorin and further developed by Bondesson.
\end{abstract}

\section{Introduction}

A key problem in Bayesian nonparametric inference is the definition of a prior distribution on the space of all probability measures. Starting from the papers by Ferguson (1973) and Freedman (1963), in which the celebrated Dirichlet process has been introduced, various approaches for constructing random probability measures, whose distribution acts as a nonparametric prior, have been undertaken. They all aim at providing generalizations of the Dirichlet process. Among them we mention the neutral-to-the-right random probability measures due to Doksum (1974), which are obtained via an exponential transformation of an increasing process with independent increments, and the Pólyatree-priors thoroughly studied by Mauldin, Sudderth and Williams (1992) and Lavine (1992), which arise by considering suitable urn schemes on trees of nested partitions. Moreover, it is well-known that the Dirichlet process can be defined by normalizing the increments of a gamma process. The idea of constructing random probability measures by means of a normalization procedure has been exploited and developed in a variety of contexts not closely related to Bayesian inference. Indeed it has found many interesting applications: Kingman (1975) and Janson (2001) for storage problems and applications to computer science; Ewens and Tavaré (1995) and Grote and Speed (2002) for population genetics; Engen (1978) and McCloskey (1965) for ecology; Derrida (1981) and Ruelle (1987) for statistical physics; Donnelly and Grimmett (1993) and Pitman (2002) for combinatorics and number theory; Pitman (1997) and Pitman and Yor (1997) for excursion theory.

Kingman's (1975) paper suggests that one can construct random probability measures as follows. First take the ranked points of a homogeneous Poisson process on $(0, \infty)$, say $\Delta_{1}>\Delta_{2}, \ldots$, such that their sum $\sum_{i=1}^{\infty} \Delta_{i}$ is finite and positive almost surely. Use these points to construct a

\footnotetext{
${ }^{1}$ AMS 2000 subject classifications. Primary 62G05; secondary 62F15.

Keywords and phrases. Bayesian Nonparametrics, Chinese restaurant process, Generalized gamma convolutions, Gibbs partitions, Poisson random measure
} 
sequence of probabilities, $\left(Q_{i}=\Delta_{i} / \sum_{l=1}^{\infty} \Delta_{l}\right)$. Independent of this sequence choose $\left(Z_{i}\right)$ to be an iid sequence of random elements of a Polish space with common distribution, say $H$. A random probability measure is then formed by $\sum_{i=1}^{\infty} Q_{i} \delta_{Z_{i}}$. Two special cases include the Dirichlet process [Ferguson (1973)] and a class of random probability measures based on the stable law discussed in for instance Pitman (1996). It is interesting to note that much of the analysis related to such quantities focuses exclusively on the behaviour of the sequence $\left(Q_{i}\right)$. Such studies were carried out for instance by Pitman, Perman and Yor (1992) and Pitman (2003). Note that because of the independence of $\left(Q_{i}\right)$ and $\left(Z_{i}\right)$, the distinctive features of different classes of random probability measures are in fact deduced exclusively from the analysis of $Q_{i}$. However with the exception of the Dirichlet process and those models based on a stable law, the analysis related to the bulk of these processes has yet to yield tractable results suitable for practical implementation in Bayesian nonparametric problems. This is due in part to the fact that the focus in Kingman (1975) and the majority of the subsequent analysis involves considerations other than Bayesian applications. Thus the issues of general tractability, in terms of their possible usage in a Bayesian context, raised by Adrian Smith and others in Kingman (1975), remains open.

In this paper we provide an analysis, for a larger class of models, with a view toward practical implementation and a better theoretical understanding of such models in a Bayesian context. Using the Dirichlet process as a benchmark, such an analysis requires a suitable description of the posterior distribution, analogous to that given by Ferguson (1973). Additionally, noting the success of the Dirichlet process in complex mixture models, we find tractable analogues of the Blackwell-MacQueen Pólya Urn and accompanying Chinese restaurant distribution which is related to the Ewens sampling formula derived by Ewens (1972) and Antoniak (1974). This then paves the way for the description of implementable MCMC and SIS computational procedures to approximate efficiently posterior quantities in applications such as hierarchical mixture modeling. Also, the exchangeable marginal distribution is equivalent to the notion of the moment measures of a random probability measure and hence is basic to the understanding of its theoretical properties and to the calculation of higher order moments. Of course, as a natural byproduct, our analysis has implications in the related nonBayesian contexts described above. Our methodology follows the paradigm laid out in James (2005a, 2002). James (2005a) points out that, in analogy to the use of classical Bayes rule, one often has to introduce additional refinements to obtain the most tractable forms. Here we show that such refinements are achieved by the use of an important exploitation of a latent structure derived from the gamma identity.

We shall actually analyze a richer and more complex generalization of the model suggested by Kingman (1975), which have recently arisen in a Bayesian context. Regazzini, Lijoi and Prünster (2003) consider random probability measures obtained by a normalization of suitably time-changed increasing processes with independent and not necessarily stationary increments. Their interest was primarily the formidable problem of the determining the distribution of mean functionals. That is a generalization of the important body of work initiated by Cifarelli and Regazzini (1990). James (2002), using an approach closely connected to Perman, Pitman and Yor (1992), considers a more general $h$-biased variation of the random probability measures which allows for an extension to arbitrary Polish spaces. The constructions coincide on Euclidean spaces when $h(s)=s$, for $s \in(0, \infty)$. In any case, such models can also be represented in terms of corresponding $\left(Q_{i}\right)$ and $\left(Z_{i}\right)$. However, now these two sequences are not necessarily independent, meaning one cannot just analyze the $\left(Q_{i}\right)$. Moreover the $\left(Q_{i}\right)$ are now generated by an $h$-biased structure.

We also provide a more specific analysis of three classes of random probability measures which are somehow connected to the Dirichlet process, and hence inherit many of its desirable features, but are otherwise much more general. Specifically we define and examine a class of dependent Dirichlet processes, a class of random probability connected to the beta process. Lastly, using the $h$-biased framework, we construct random probability measures based on the theory of Generalized Gamma Convolutions (GGC), initiated by Thorin (1977, 1978) and provide a detailed analysis. Some interesting features of this construction, is that we are able to embed a very large class 
of random probability measures within a tractable framework of models which are extensions of Dirichlet process mean functionals. More precisely, these are models derived from a gamma process with a shape measure which is sigma finite and therefore has possibly infinite total mass. Our construction includes, for instance the stable law random probability measure and hence the entire class of the two-parameter Poisson-Dirichlet process. Moreover our results and discussions are strongly suggestive of a more synergistic interplay between the theory of GGC and the study of the laws of extensions of mean functionals of the Dirichlet process.

We next describe the formal construction and discuss the key features of our analysis.

\section{$1.1 \quad$ Preliminaries}

Let $N$ denote a Poisson random measure on an arbitrary Polish Space $\mathscr{S} \times \mathscr{X}$, with mean intensity

$$
\mathbb{E}[N(d s, d x)]=\nu(d s, d x) .
$$

Denote the law of $N$ as $\mathbb{P}(\cdot \mid \nu)$ with $\mathbb{E}[\cdot \mid \nu]$ denoting expectation with respect to $\mathbb{P}$. The distribution of $N$ is characterized by the Laplace functional

$$
\mathbb{E}\left[\mathrm{e}^{-N(g)}\right]=\exp \left(-\int_{\mathscr{X} \times \mathscr{S}}\left(1-\mathrm{e}^{-g(s, x)}\right) \nu(\mathrm{d} s, \mathrm{~d} x)\right)
$$

where $N(g)=\int_{\mathscr{S} \times \mathscr{X}} g(s, x) N(d s, d x)$ and $g$ is any positive function. Throughout, as in Daley and Vere-Jones (1986), we have that $N$ and all related functionals take their values in the space of boundedly finite measures on $\mathscr{S} \times \mathscr{X}$, say $\mathbb{M}$. A measure, say $\Lambda$, is boundedly finite if for each bounded set $A, \Lambda(A)<\infty$. According to the decomposition of the intensity measure, we distinguish the two following cases:

(i) if $\nu(\mathrm{d} s, \mathrm{~d} x)=\rho(\mathrm{d} s) H(\mathrm{~d} x)$ we say that $N$ and its related functionals are homogeneous

(ii) if $\nu(\mathrm{d} s, \mathrm{~d} x)=\rho(\mathrm{d} s \mid x) \eta(\mathrm{d} x)=F_{0}(\mathrm{~d} x \mid s) \rho(\mathrm{d} s), N$ and its related functionals are non-homogeneous.

Where $H$ and $F_{0}$ are probabilities on $\mathscr{X}, \eta$ is a finite measure on $\mathscr{X}$. The quantity, $\rho(d s \mid x)$ is an inhomogeneous Lévy measure for each fixed $x$. The quantity,

$$
\rho(d s)=\int_{\mathscr{X}} \rho(d s \mid x) \eta(d x)
$$

is a then a homogeneous Lévy measure on $\mathscr{S}$. Let $\left(J_{i}, Z_{i}\right)$ denote the sequence of points of $N$ on $\mathscr{S} \times \mathscr{X}$. Using the decomposition $F_{0}(d x \mid s) \rho(d s)$, it follows that now for each $i$, the conditional distribution of $Z_{i}$ given $J_{i}$ is

$$
\mathbb{P}\left(Z_{i} \in d x \mid J_{1}, J_{2}, \ldots\right):=\mathbb{P}\left(Z_{i} \in d x \mid J_{i}\right)=F_{0}\left(d x \mid J_{i}\right) .
$$

Moreover the marginal distribution of the sequence $\left(J_{i}\right)$ are the random points in $\mathscr{S}$ of a homogeneous Poisson random measure with mean intensity $\rho(d s)$. See for instance Resnick (1987, section 3.3.2).

Let $h$ denote a strictly positive function on $\mathscr{S}$. Now define a random measure on $\mathscr{X}$, which is representable in distribution as,

$$
\mu(d x)=\int_{\mathscr{S}} h(s) N(d s, d x)
$$

such that its total mass $T:=\mu(\mathscr{X})=\sum_{i=1}^{\infty} h\left(J_{i}\right)$, is strictly positive and almost surely finite. This happens if $\nu(\mathscr{S} \times \mathscr{X})=+\infty$ and the Laplace transform

$$
\phi(\lambda)=\mathbb{E}\left[\mathrm{e}^{-\lambda T}\right]=\mathrm{e}^{-\psi(\lambda)}
$$


where

$$
\psi(\lambda):=\int_{\mathscr{X} \times \mathscr{S}}\left(1-\mathrm{e}^{-\lambda h(s)}\right) \nu(\mathrm{d} s, \mathrm{~d} x)
$$

is finite for any positive $\lambda$. We now can define a class of random probability measures on $\mathscr{X}$ representable in distribution as,

$$
P(d x)=\frac{\int_{\mathscr{S}} h(s) N(d s, d x)}{T}=\frac{\mu(d x)}{T}=\sum_{i=1}^{\infty} Q_{i} \delta_{Z_{i}}=\sum_{i=1}^{\infty} \frac{h\left(J_{i}\right)}{T} \delta_{Z_{i}}
$$

We will call the class of $P$ defined as (2), normalized random measures or NRMs. In Regazzini et al. (2003) an analogous construction, with $\mathscr{S}=(0,+\infty)$ and $\mathscr{X}=\mathbb{R}$, has been developed by normalizing the increments of an increasing additive process.

REMARK 1. Note that the generality of $\mathscr{S}$ allows for quite complex spaces. For example, one could take $\mathscr{S}$ to be the space of probability measures. Or $\mathscr{S}$ could denote the space containing the sample paths of Brownian Motion or more general stochastic processes. See Perman, Pitman and Yor (1992) for an example involving excursion spaces of Markov processes.

REMARK 2. Pitman (2003) provides an important extension of the class of homogeneous NRM, defined by $h(s)=s \in(0, \infty)$, which can be defined as follows. For a homogeneous $\rho$, denote the law of the sequence $\left(J_{i} / T\right)$ as $P K(\rho)$ Furthermore, define the law of $\left(J_{i} / T\right) \mid T=t$, as $P K(\rho \mid t)$, where $T$ has density $f_{T}(t)$. Then this class generates the class of laws on $\left(J_{i} / T\right)$, given by $P K\left(\rho ; \gamma_{T}\right)=$ $\int_{0}^{\infty} P K(\rho \mid t) \gamma_{T}(d t)$, where $\gamma_{T}$ is some density of $T$. An interesting special case is the choice of $\gamma_{T}(t) \propto t^{-q} f_{T}(t)$, which, with the exception of the Dirichlet process, yields the two-parameter Poisson Dirichlet process when $f_{T}$ is the density of the stable law of index $0<\alpha<1$. The idea of conditioning on $T$ is discussed briefly in Kingman (1975). In order to capture models like this and more general processes we can instead work with a weighted Poisson law of the type $g(N) \mathbb{P}(d N \mid \nu)$ where we assume that $\int_{\mathbb{M}} g(N) \mathbb{P}(d N \mid \nu)=1$. Formal details are given in section 8.3.

\section{Posterior Analysis}

Similar to Ferguson (1973) we shall consider the classical setup. Let $\left(X_{n}\right)_{n \geq 1}$ be a sequence of $\mathscr{X}-$ valued exchangeable random elements, i.e. such that for any $n \geq 1$ the $X_{1}, \ldots, X_{n}$ are, conditional on $P$, iid with common distribution $P$. Suppose, also, that $P$ is an NRM. This yields a description of the joint distribution of $(\mathbf{X}, P)$. We are interested in obtaining its description in terms of a posterior distribution of $P \mid \mathbf{X}$ and the (exchangeable) marginal distribution of $\mathbf{X}$, say $\mathscr{M}$. Since the law of $P$ is dominated by the law of $N$ it follows that the posterior distribution of $P \mid \mathbf{X}$ is determined by the posterior distribution of $N \mid \mathbf{X}$. Moreover $\mathscr{M}$ can be expressed as,

$$
\mathscr{M}\left(d x_{1}, \ldots, d x_{n}\right)=\int_{\mathbb{M}}\left[\prod_{i=1}^{n} P\left(d x_{i}\right)\right] \mathbb{P}(d N \mid \nu)=\int_{\mathbb{M}} T^{-n}\left[\prod_{i=1}^{n} \int_{\mathscr{S}} h\left(s_{i}\right) N\left(d s_{i}, d x_{i}\right)\right] \mathbb{P}(d N \mid \nu)
$$

and is the general analogue of the Blackwell-MacQueen Pólya Urn distribution. We recall from James (2005a) that there is a one to one correspondence between $\mathbf{X}$ and ( $\mathbf{Y}, \mathbf{p})$, where using notation similar to Lo (1984), $\mathbf{Y}=\left(Y_{1}, \ldots, Y_{n(\mathbf{p})}\right)$ denotes the distinct values of $\mathbf{X}$ and $\mathbf{p}=\left\{C_{1}, \ldots, C_{n(\mathbf{p})}\right\}$ stands for a partition of the integers $\{1, \ldots, n\}$ of size $n(\mathbf{p}) \leq n$ recording which observations are equal. The number of elements in the $j$-th cell, $C_{j}:=\left\{i: X_{i}=Y_{j}\right\}$, of the partition is indicated by $e_{j}$, for $j=1, \ldots, n(\mathbf{p})$, so that $\sum_{j=1}^{n(\mathbf{p})} e_{j}=n$. When it is necessary to emphasize a further dependence on $n$, we will also use the notation $e_{j, n}:=e_{j}$. It follows that the marginal distribution of $\mathbf{X}$ can be expressed in terms of a conditional distribution of $\mathbf{X} \mid \mathbf{p}$, which is the same as a conditional distribution of the unique values $\mathbf{Y} \mid \mathbf{p}$, and the marginal distribution of $\mathbf{p}$. The marginal distribution of $\mathbf{p}$, denoted as $\pi(\mathbf{p})$ or $p\left(e_{1}, \ldots, e_{n(\mathbf{p})}\right)$, is an exchangeable partition probability function $(\mathrm{EPPF})$. 
REMARK 3. A detailed general discussion of the EPPF concept may be found in Pitman (2002). A discussion of its role in a Bayesian context pertinent to our homogeneous models, which are a special case of species sampling models, may be deduced from Pitman (1996) and Ishwaran and James (2003a). Its role for inhomogeneous models is a bit different. See James (2005a) for the role of the EPPF, and that of $\mathscr{M}$, in this more general Bayesian context. The most well-known EPPF is the Chinese restaurant distribution associated with the Dirichlet process.

\subsection{Posterior Distributions}

Now, similar to Perman, Pitman and Yor (1992, section 4) let, $N_{n(\mathbf{p})}:=N-\sum_{j=1}^{n(\mathbf{p})} \delta_{J_{j, n}, Y_{j}}$ denote a random measure after the first $n(\mathbf{p})$ pairs of unique points $\left(J_{j, n}, Y_{j}\right)$ in $\mathscr{S} \times \mathscr{X}$ are picked from $N$ by $h$-biased sampling. Define $N_{0}:=N$ and note that the law of $N_{n(\mathbf{p})}$, depends on the random variable $n(\mathbf{p})$. For each $n$ it follows that $N=N_{n(\mathbf{p})}+\sum_{j=1}^{n(\mathbf{p})} \delta_{J_{j, n}, Y_{j}}$. From this one may define

$$
\mu_{n(\mathbf{p})}=\mu-\sum_{j=1}^{n(\mathbf{p})} h\left(J_{j, n}\right) \delta_{Y_{j}} \quad \text { and } \quad T_{n(\mathbf{p})}=\mu_{n(\mathbf{p})}(\mathscr{X})=T-\sum_{j=1}^{n(\mathbf{p})} h\left(J_{j, n}\right)
$$

Now, crucial to our exposition, for each $n$ define the random variable $U_{n}=\Gamma_{n} / T$, where $\Gamma_{n}$ denotes a gamma random variable with shape $n$ and scale 1 which is independent of $N$ and hence $T$. Throughout our exposition $\Gamma_{n}$ will always be taken to be independent of every random variable except for $U_{n}$. It will be shown in the appendix, that the appearance of $U_{n}$ arises from an application of the Gamma identity,

$$
T^{-n}=\frac{1}{\Gamma(n)} \int_{0}^{\infty} u^{n-1} \mathrm{e}^{-u T} d u
$$

It turns out that importantly, $U_{n}$ is also intimately connected to the real inversion formula for the Laplace transform. This is discussed in Feller (1971, VII.6) and plays a prominent role in the analysis of infinite-divisibility by Thorin $(1977,1978)$ and Bondesson $(1979,1992)$. We will discuss some features of this in the forthcoming sections.

Define for each integer $l$, cumulants,

$$
\tau_{l}(u \mid y)=\int_{\mathscr{S}}[h(s)]^{l} \mathrm{e}^{-u h(s)} \rho(d s \mid y) \text { and } \kappa_{l}(u)=\int_{\mathscr{S}}[h(s)]^{l} \mathrm{e}^{-u h(s)} \rho(d s)
$$

Define conditional distributions of the $\left(J_{j, n}\right)$ given $\left(U_{n}, \mathbf{X}\right)$, as

$$
\mathbb{P}\left(J_{j, n} \in d s \mid Y_{j}, u\right)=\frac{[h(s)]^{e_{j}} \mathrm{e}^{-u s} \rho\left(d s \mid Y_{j}\right)}{\tau_{e_{j}}\left(u \mid Y_{j}\right)}, \text { for } j=1, \ldots, n(\mathbf{p})
$$

Note that, conditional on $U_{n}$, each $J_{j, n}$ only depends upon $\mathbf{X}$ through $\left(e_{j}, Y_{j}\right)$. In the homogeneous case their distributions no longer depend upon $Y_{j}$ and correspond to the distribution of $\left(J_{j, n}\right) \mid U_{n}, \mathbf{p}$ expressible as $\mathbb{P}\left(J_{j, n} \in d s \mid u\right) \propto[h(s)]^{e_{j}} \mathrm{e}^{-u s} \rho(d s)$. We note further that

$$
\mathbb{E}\left[h\left(J_{j, n}\right) \mid u, \mathbf{X}\right]=\frac{\tau_{1+e_{j, n}}\left(u \mid Y_{j}\right)}{\tau_{e_{j, n}}\left(u \mid Y_{j}\right)} \quad \text { and } \quad \mathbb{E}\left[h\left(J_{j, n}\right) \mid u, \mathbf{p}\right]=\frac{\kappa_{1+e_{j, n}}(u)}{\kappa_{e_{j, n}}(u)} .
$$

We now use these variables to describe the relevant posterior distributions and related quantities.

Theorem 2.1 Let $P$ denote a NRM defined by the Poisson random measure $N$ with mean intensity $\nu(d s, d x)=\rho(d s \mid x) \eta(d x)$. Let $\mathbf{X}=\left(X_{1}, \ldots, X_{n}\right)$ denote a vector of random elements on a Polish space such that $X_{1}, \ldots, X_{n} \mid P$ are iid $P$, then the following results hold. 
(i) The posterior distribution of $N$, given $\left(U_{n}, \mathbf{X}\right)$, coincides with the conditional distribution of the random measure $N_{n}^{*}=N_{n(\mathbf{p})}+\sum_{j=1}^{n(\mathbf{p})} \delta_{\left(J_{j, n}, Y_{j}\right)}$, where conditional on $U_{n}=u$ and $\mathbf{X}, N_{n(\mathbf{p})}$ is a Poisson random measure with intensity

$$
\nu_{u}(\mathrm{~d} s, \mathrm{~d} x)=e^{-u h(s)} \rho(\mathrm{d} s \mid x) \eta(\mathrm{d} x)=e^{-u h(s)} F_{0}(d x \mid s) \rho(\mathrm{d} s),
$$

not depending on $\mathbf{X}$, except through $U_{n}$.

(ii) Additionally, the $\left(J_{j, n}\right)$ are, conditional on $\left(U_{n}, \mathbf{X}\right)$, independent of $N_{n(\mathbf{p})}$ and are mutually independent with each $J_{j, n}$ having the distribution, specified in (4)

(iii) The posterior density of $U_{n} \mid \mathbf{X}$, is equivalent to the density of $\Gamma_{n} /\left[T_{n(\mathbf{p})}+\sum_{j=1}^{n(\mathbf{p})} h\left(J_{j, n}\right)\right] \mid \mathbf{X}$, which is $f_{U_{n}}(u \mid \mathbf{X}) \propto u^{n-1} \mathrm{e}^{-\psi(u)} \prod_{j=1}^{n(\mathbf{p})} \tau_{e_{j}}\left(u \mid y_{j}\right)$. Similarly, the density of $U_{n} \mid \mathbf{p}$ is $f_{U_{n}}(u \mid \mathbf{p}) \propto$ $u^{n-1} \mathrm{e}^{-\psi(u)} \prod_{j=1}^{n(\mathbf{p})} \kappa_{e_{j}}(u)$.

Since $\mu$ and $P$ are functionals of $N$ the next two results follow immediately.

Proposition 2.1 The posterior distribution of $\mu \mid U_{n}, \mathbf{X}$ is equivalent to the conditional distribution, given $U_{n}, \mathbf{X}$, of the random measure $\mu_{n}^{*}(d x)=\int_{0}^{\infty} s N_{n}^{*}(d s, d x)=\mu_{n(\mathbf{p})}(d x)+\sum_{j=1}^{n(\mathbf{p})} h\left(J_{j, n}\right) \delta_{Y_{j}}(d x)$, where conditional on $U_{n}$ and $\mathbf{X}, \mu_{n(\mathbf{p})}(d x):=\int_{0}^{\infty} s N_{n(\mathbf{p})}(d s, d x)$ is a completely random measure with Lévy measure specified in (5). This implies that the density of $T_{n(\mathbf{p})} \mid U_{n}=u, \mathbf{X} i s f_{T_{n(\mathbf{p})}}(t \mid u)=$ $e^{-u t} e^{\psi(u)} f_{T}(t)$.

Proposition 2.2 The posterior distribution of $P \mid \mathbf{X}$, is equivalent to the conditional distribution of the random probability measure,

$$
P_{n}^{*}(d y)=R_{n(\mathbf{p})} P_{n(\mathbf{p})}(d y)+\sum_{j=1}^{n(\mathbf{p})} Q_{j, n} \delta_{Y_{j}}(d y)
$$

where $P_{n(\mathbf{p})}(d x)=\mu_{n(\mathbf{p})}(d x) / T_{n(\mathbf{p})}, R_{n(\mathbf{p})}=T_{n(\mathbf{p})} / T=1-\sum_{j=1}^{n(\mathbf{p})} Q_{j, n}$ and $Q_{j, n}=h\left(J_{j, n}\right) / T$ for $j=1, \ldots, n(\mathbf{p})$. The distribution of all quantities, given $\left(U_{n}, \mathbf{X}\right)$, is specified by Theorem 2.1.

We now provide an initial description of $\mathscr{M}$.

Proposition 2.3 The exchangeable marginal distribution $\mathscr{M}$ of the observations $\mathbf{X}$ can be represented as

$$
\mathscr{M}\left(\mathrm{d} x_{1}, \ldots, \mathrm{d} x_{n}\right)=\frac{1}{\Gamma(n)}\left\{\int_{0}^{+\infty} u^{n-1}\left[\prod_{j=1}^{n(\mathbf{p})} \tau_{e_{j}}\left(u \mid y_{j}\right)\right] \mathrm{e}^{-\psi(u)} \mathrm{d} u\right\} \prod_{j=1}^{n(\mathbf{p})} \eta\left(\mathrm{d} y_{j}\right)
$$

The corresponding EPPF is

$$
p\left(e_{1}, \ldots, e_{n(\mathbf{p})}\right)=[\Gamma(n)]^{-1} \int_{0}^{+\infty} u^{n-1}\left[\prod_{j=1}^{n(\mathbf{p})} \kappa_{e_{j}}(u)\right] \mathrm{e}^{-\psi(u)} \mathrm{d} u .
$$

REMARK 4. In the case where $h(s)=s \in(0, \infty)$, the EPPF appearing in Proposition 2.3 was first obtained by Pitman (2003, Corollary 6). Note that EPPF's appearing in Proposition 2.3 are examples of infinite EPPF's. In the next section we shall show that they may be represented as a mixture of tractable finite EPPF's. The distinctions between finite and infinite EPPF's are described in Pitman (2002, Section 2). 


\section{Analysis of the exchangeable marginal distribution $\mathscr{M}$}

In this section we present a simpler description of the marginal distribution $\mathscr{M}$ and the corresponding EPPF. Such a description facilitates its practical implementation and indeed has theoretical implications as well. Note that ideally one would like an EPPF of the form

$$
p\left(e_{1}, \ldots, e_{n(\mathbf{p})}\right)=v_{n, n(\mathbf{p})} \prod_{j=1}^{n(\mathbf{p})} w_{e_{j}} .
$$

where here $v_{n, n(\mathbf{p})}$ is a positive quantity only depending on $n$ and $n(\mathbf{p})$, and the $w_{e_{j}}$ are a sequence of positive numbers each only depending on $e_{j}$ for $j=1, \ldots, n(\mathbf{p})$. One aspect of such a representation is that it is easily sampled according to variations of general Chinese restaurant processes. Pitman (2002) refers to such EPPF as having Gibbs form. However, it is known [see Pitman (2002), Theorem 42, p. 81] that the only infinite EPPF admitting such a representation are the EPPF's derived from a Dirichlet process and those derived from a Stable law of index $0<\alpha<1$. Among them, we mention the two-parameter Dirichlet process and the generalized gamma class of processes. Here again we show that the appropriate usage of the random variable $U_{n}$, leads to tractable descriptions of the $\mathscr{M}$ and the EPPF.

These simplifications are deduced from the following joint distribution of $\left(\mathbf{p}, T_{n(\mathbf{p})}, U_{n}, \mathbf{Y}\right)$ given by $\mathbb{P}\left(\mathbf{p}, T_{n(\mathbf{p})} \in \mathrm{d} z, U_{n} \in \mathrm{d} u, \mathbf{Y} \in \mathrm{d} \mathbf{y}\right)$ equal to,

$$
\frac{1}{\Gamma(n)} u^{n-1} \mathrm{e}^{-u z} f_{T}(z) \mathrm{d} u \mathrm{~d} z \prod_{j=1}^{n(\mathbf{p})} \tau_{e_{j}}\left(u \mid y_{j}\right) \eta\left(\mathrm{d} y_{j}\right)
$$

This distribution appears naturally in our derivation of the posterior distribution given in the appendix. Now, for fixed $u>0$ and $\mathbf{p}$, let

$$
\mathbb{P}\left(Y_{j} \in d y \mid U_{n}=u, \mathbf{p}\right):=H_{j, n}(\mathrm{~d} y \mid u)=\frac{\tau_{e_{j, n}}(u \mid y) \eta(\mathrm{d} y)}{\kappa_{e_{j, n}}(u)} \quad j=1, \ldots, n(\mathbf{p})
$$

Theorem 3.1 Let $\mathbf{X}$ denote the random variables with the exchangeable distribution $\mathscr{M}$ described in Proposition 2.3. Then the distribution of $\mathbf{X}$ may be described as follows.

(i) The distribution of $\mathbf{X} \mid U_{n}=u, \mathbf{p}$ is such that the unique values $Y_{1}, \ldots, Y_{n(\mathbf{p})}$ are independent with respective distributions given by (9). In the homogeneous case, it follows that $Y_{1}, \ldots, Y_{n(\mathbf{p})} \mid \mathbf{p}$ are iid with common distribution $\eta(d x) / \eta(\mathscr{X})=H(d x)$ not depending on $U_{n}$.

(ii) The distribution of $\mathbf{p} \mid U_{n}=u, \mathbb{P}\left(\mathbf{p}=\left\{C_{1}, \ldots, C_{n(\mathbf{p})}\right\} \mid U_{n}=u\right)$, is a conditional finite EPPF given by

$$
p\left(e_{1}, \ldots, e_{n(\mathbf{p})} \mid u\right)=\frac{\mathrm{e}^{-\psi(u)} \prod_{j=1}^{n(\mathbf{p})} \kappa_{e_{j}}(u)}{\int_{0}^{\infty} t^{n} \mathrm{e}^{-u t} f_{T}(t) d t} .
$$

where $E\left[T_{n(\mathbf{p})}^{n} \mid U_{n}=u\right]=\mathrm{e}^{\psi(u)} \int_{0}^{\infty} t^{n} \mathrm{e}^{-u t} f_{T}(t) d t$ That is, conditional on $U_{n}=u, \mathbf{p}$ is a finite Gibbs partition.

(iii) The marginal density of $U_{n}=\Gamma_{n} / T$ is

$$
f_{U_{n}}(u)=[\Gamma(n)]^{-1} u^{n-1} \int_{0}^{\infty} t^{n} \mathrm{e}^{-u t} f_{T}(t) d t .
$$


Proof. Statement (i) follows by applying Bayes rule to (8). Statement (iii) is straightforward. An application of Bayes rule also yields readily a description of the distribution of $\mathbf{p} \mid U_{n}=u$, where the normalizer is $\sum_{\mathbf{p}} \prod_{j=1}^{n(\mathbf{p})} \kappa_{e_{j}}(u)$. Here $\sum_{\mathbf{p}}$ stands for the sum over all partitions of the set of integers $\{1, \ldots, n\}$. The simpler form in (10) may be obtained by noting some known relationships between cumulants, partitions and moments. However, for immediate clarity one can use (8) to establish the identity $f_{U_{n}}(u)=[\Gamma(n)]^{-1} u^{n-1} \mathrm{e}^{-\psi(u)} \sum_{\mathbf{p}} \prod_{j=1}^{n(\mathbf{p})} \kappa_{e_{j}}(u)$. The result then follows by noting the form of $f_{U_{n}}(u)$ given in (iii).

The next proposition offers another description of the distribution of the unique values.

Proposition 3.1 Suppose that $\mathbf{X}$ has distribution $\mathscr{M}$. Then using the decomposition $\nu(d s, d x)=$ $F_{0}(d x \mid s) \rho(d s)$, it follows that the distribution of $Y_{1}, \ldots, Y_{n(\mathbf{p})}$ given $U_{n}, J_{1, n}, \ldots, J_{n(\mathbf{p}), n}, \mathbf{p}$ is such that the $Y_{j}$ are independent with respective distributions $\mathbb{P}\left(Y_{j} \in d y \mid J_{j, n}\right)=F_{0}\left(d y \mid J_{j, n}\right)$. That is, the conditional distributions only depend on $U_{n}$ through the $\left(J_{j, n}\right)$

REMARK 5. The above results demonstrate the important role that $U_{n}$ plays in simplifying the above quantities. In effect, conditioning on $U_{n}$, reveals conditional likelihoods that have exponential form. This exponential form bears resemblance to those appearing in the posterior analysis of neutral to the right processes and the Levy moving average models discussed in James (2005a). Models of this type are most amenable to the direct application of James (2005a, Proposition 2.3). It is then not surprising that one may notice some similarities between our posterior characterizations and those described by Doskum (1974), Ferguson (1974), Hjort(1990) and Kim (1999) for NTR models. We point out however that these models are otherwise very different, see James (2005a,2005c).

REMARK 6. We note further that conditioning on $T$, instead of $U_{n}$, does not lead to simplified expressions. See however Pitman (2003) for important interpretations of conditioning on $T$ in the stable case.

We close this section by showing how $U_{n}$ is related to what is called the real inversion formula for the Laplace transform as described in Bondesson (1992, eq. (6.2.1), p. 92)[see Feller (1971,VII)]. In some sense it shows that $U_{n}$ is asymptotically sufficient for $T$. The result below follows directly from Feller (1971, VII).

Proposition 3.2 Let $Y_{n}=n / U_{n}=n T / \Gamma_{n}$. Then the $p d f$ of $Y_{n}$ is given by

$$
f_{Y_{n}}(y)=\frac{1}{\Gamma(n+1)}\left(\frac{n}{y}\right)^{n+1} \int_{0}^{\infty} e^{-n t / y} t^{n} f_{T}(t) d t=\frac{(-1)^{n}}{n !}\left(\frac{n}{y}\right)^{n+1} \phi^{(n)}(n / y)
$$

where $\phi^{(n)}$ denotes the $n$-th derivative of $\phi$. Additionally it follows that $f_{Y_{n}}(t)$ converges to $f_{T}(t)$ uniformly in every finite interval, as $n \rightarrow \infty$. Hence (11) is an inversion formula for the Laplace transform of $T$

REMARK 7. Comparing (10) with (7) shows that conditional on $U_{n}$, the distribution of $\mathbf{p}$, is a finite EPPF of Gibbs form. In particular for fixed $u$, we see that in this case,

$$
v_{n, n(\mathbf{p})}=E\left[T_{n(\mathbf{p})}^{n} \mid U_{n}=u\right]=\mathrm{e}^{\psi(u)} \int_{0}^{\infty} t^{n} \mathrm{e}^{-u t} f_{T}(t) d t
$$

which importantly does not depend on $n(\mathbf{p})$, and $w_{e_{j}}=\kappa_{e_{j, n}}(u)$. This has many interesting consequences, of which we shall highlight a few in the forthcoming sections. 


\subsection{Distributional results and moment formulae for complex functionals}

The next result gives an expression for the distribution of $n(\mathbf{p})$ given $U_{n}$.

Proposition 3.3 Let $n_{1} \geq n_{2} \ldots \geq n_{k} \geq 1$ denote an ordering (composition) of the corresponding $\left(e_{1}, \ldots, e_{k}\right)$. Recall that $n(\mathbf{p})$ represents the number of unique values of $\left(X_{1}, \ldots, X_{n}\right)$. Then under the distribution $\mathscr{M}$, the conditional distribution of $n(\mathbf{p})$ given $U_{n}=u$, is given by

$$
\mathbb{P}\left(n(\mathbf{p})=k \mid U_{n}=u\right)=\frac{\mathrm{e}^{-\psi(u)}}{\int_{0}^{\infty} t^{n^{2}} \mathrm{e}^{-u t} f_{T}(t) d t} \frac{n !}{k !} \sum_{\left(n_{1}, \ldots, n_{k}\right)} \prod_{j=1}^{k} \frac{\kappa_{n_{j}}(u)}{n_{j} !}
$$

for $k=1, \ldots, n$. Where the sum corresponds to the sum over all compositions $\left(n_{1}, \ldots, n_{k}\right)$ of $n$ of size $n(\mathbf{p})=k$.

The fact that $\mathbf{p} \mid U_{n}$ is a finite Gibbs partition allows us to apply Pitman (2002, eq. 98) [see also Kolchin (1986)] to immediately deduce the following generalization of the Ewens sampling formula, Ewens (1972), and equivalently Antoniak (1974, Proposition 3).

Proposition 3.4 Define a random vector $\left(\left|\Pi_{i, n}\right|\right.$, for $\left.1 \leq i \leq n\right)$ of non-negative counts by $\left|\Pi_{i, n}\right|=$ $\sum_{j=1}^{n} I\left(\left|C_{j, n}\right|=i\right)$ for $i=1, \ldots, n$. Where specifically, $\left|\Pi_{i, n}\right|$ denotes the number of cells of size $i$. The distribution of $\left(\left|\Pi_{i, n}\right|\right.$, for $\left.1 \leq i \leq n\right)$ given $U_{n}=u$, can be represented as

$$
\mathbb{P}\left(\left|\Pi_{j, n}\right|=m_{j}, 1 \leq j \leq n \mid u\right)=\frac{n ! \mathrm{e}^{-\psi(u)}}{\int_{0}^{\infty} t^{n} \mathrm{e}^{-u t} f_{T}(t) d t} \prod_{j=1}^{n}\left(\frac{\kappa_{j}(u)}{j !}\right)^{m_{j}} \frac{1}{m_{j} !}
$$

where $\sum_{j=1}^{n} m_{j}=k$ and $\sum_{j=1}^{n} j m_{j}=n$. Equivalently 12) is the conditional distribution, given $U_{n}$, of the number of values of $\left(X_{1}, \ldots, X_{n}\right)$ appearing 1 time, 2 times etc, corresponding to the numbers $\left(m_{1}, \ldots, m_{n}\right)$, when $\mathbf{X}$ has distribution $\mathscr{M}$.

The recognition that $\mathscr{M}$ is the $n-$ th moment measure of $P$ allows one to obtain easily otherwise complex expressions for moments of functionals of $P$. The discussion in Ishwaran and James (2003a, section 3.2) combined with Proposition 2.1 leads to the following formula. Recall that $\sum_{\mathbf{p}}$ denotes the sum over all partitions of the set $\{1, \ldots, n\}$.

Proposition 3.5 Suppose that $g_{1}, \ldots, g_{n}$ are real-valued functions on $\mathbf{X}$ and define the functionals $P\left(g_{i}\right)=\int_{\mathcal{X}} g_{i}(x) P(d x)$ for $i=1, \ldots n$. Assume that for each $n, E\left[\prod_{i=1}^{n}\left(P\left(g_{l}\right)\right)\right]<+\infty$.

(i) Then, $\mathbb{E}\left[\prod_{l=1}^{n} P\left(g_{l}\right)\right]$ is equal to,

$$
\int_{0}^{\infty}\left[\sum_{\mathbf{p}} \pi(\mathbf{p} \mid u) \prod_{j=1}^{n(\mathbf{p})} \int_{\mathbf{X}}\left[\prod_{i \in C_{j}} g_{i}(y)\right] H_{j, n}(\mathrm{~d} y \mid u)\right] f_{U_{n}}(u) \mathrm{d} u .
$$

(ii) For integers $n_{1}, \ldots, n_{q}$ chosen such that $\sum_{i=1}^{q} n_{i}=n$ for an integer $q \leq n$, it follows that, $E\left[\prod_{l=1}^{q}\left(P\left(g_{l}\right)\right)^{n_{l}}\right]$ is equal to

$$
\int_{0}^{\infty}\left[\sum_{\mathbf{p}} \pi(\mathbf{p} \mid u) \prod_{j=1}^{n(\mathbf{p})} \int_{\mathbf{X}}\left[\prod_{l=1}^{q}\left[g_{l}(y)\right]^{e_{j, l}}\right] H_{j, n}(\mathrm{~d} y \mid u)\right] f_{U_{n}}(u) \mathrm{d} u .
$$

Where $e_{j, l}$ denotes the number of indices in $C_{j}$ associated with $g_{l}$. 
REMARK 8. It is interesting to note that all our results conditioned on $U_{n}$, contain the known unconditional results for the Dirichlet process. This is because the Dirichlet process is independent of $U_{n}$. To see this, note that the Dirichlet process with total mass $\theta$, corresponds to the choice of $\rho(d s)=\theta s^{-1} \mathrm{e}^{-s} d s$. It follows that for each $j$ that $\kappa_{j}(u)=\theta(1+u)^{-j} \Gamma(j)$ and $E\left[T_{n(\mathbf{p})} \mid u\right]=$ $(1+u)^{-n}[\Gamma(\theta) / \Gamma(\theta+n)]$. Aditionally the $f_{U_{n}}(u \mid \mathbf{p}):=f_{U_{n}}(u) \propto u^{n-1}(1+u)^{-(n+\theta)}$. That is $U_{n}=\Gamma_{n} / T$ is a gamma-gamma random variable independent of $\mathbf{X}$. Equivalently $1 /\left(1+U_{n}\right)$ is a $\operatorname{Beta}(\theta, n)$ random variable. Hence, (12), specializes to

$$
\mathbb{P}\left(\left|\Pi_{j, n}\right|=m_{j}, 1 \leq j \leq n \mid u\right)=\frac{n !}{\prod_{i=1}^{n}(\theta+i-1)} \prod_{j=1}^{n}\left(\frac{\theta}{j}\right)^{m_{j}} \frac{1}{m_{j} !}
$$

This equates to the Ewens sampling formula derived by Ewens (1972), which is equivalent to the result in Antoniak (1974, Proposition 3) describing the number of values of $\left(X_{1}, \ldots, X_{n}\right)$ appearing 1 time, 2 times etc, corresponding to the numbers $\left(m_{1}, \ldots, m_{n}\right)$. Additionally, note that (10) becomes,

$$
\operatorname{PD}(\mathbf{p} \mid \theta):=\frac{\theta^{n(\mathbf{p})} \prod_{j=1}^{n(\mathbf{p})}\left(e_{j}-1\right) !}{\prod_{i=1}^{n}(\theta+i-1)}
$$

which is the variant of Ewens sampling formula, often called the Chinese restaurant process. [See Pitman (2002, p. 60) and Ishwaran and James (2003a)]. The calculations for the Dirichlet process involving $U_{n}$ may be found in James $(2005 \mathrm{~b})$, where it is shown that $U_{n}$ and its variants still play a significant role.

\section{Mixture models}

In terms of statistical applications, owing to the success of the Dirichlet process, one of the most fruitful ways for exploiting NRM's is their potential use as basic building blocks in hierarchical mixture models. In this setting, $X_{1}, \ldots, X_{n}$ are missing values which capture the clustering structure within the data. This class of models was first introduced, for the Dirichlet process, by Lo (1984) and later popularized by the development of suitable MCMC techniques in Escobar and West (1995). See Dey, Müller and Sinha (1998) and Ishwaran and James (2001, 2003a,b) for subsequent developments. Recently, mixtures of Dirichlet process have been generalized to mixtures of stick-breaking priors in Ishwaran and James (2001, 2003b) and random measures driven by increasing additive processes in Nieto-Barajas, Prünster and Walker (2004). A recent example of application of this class is provided in Lijoi, Mena and Prünster (2004) where the clustering behaviour is modeled according to a normalized inverse Gaussian process. Ishwaran and James (2003a) also introduce a general class of species sampling mixture models and describe various algorithms for efficient implementation. See also Hoff (2003, section 4) for an interesting use of the Dirichlet process mixture model framework. Those ideas naturally extend to models based on the NRM's.

We first recall the model as set up by Lo (1984). Suppose $\{f(\cdot \mid x): x \in \mathscr{X}\}$ is a family of non-negative kernels defined on a Polish space $\mathbf{W}$ such that $\int_{\mathbf{W}} f(w \mid x) \lambda(\mathrm{d} w)=1$ for any $x$ in $\mathscr{X}$ and for some $\sigma$-finite measure $\lambda$. Next, let $\mathbf{W}=\left(W_{1}, \ldots, W_{n}\right)$ be a vector of $\mathbb{W}$-valued random elements such that, given $X_{1}, \ldots, X_{n}$ from a NRMI $P$, they are independent and $W_{j}$ admits density, with respect to $\lambda, f\left(\cdot \mid X_{j}\right)$. This is the same as supposing that $W_{1}, \ldots, W_{n}$ are exchangeable draws from the random density $\tilde{f}(\cdot)=\int_{\mathbf{X}} f(\cdot \mid x) P(\mathrm{~d} x)$. One is naturally interested in the determination of the distribution of the posterior density $\tilde{f}$, given the observations $\mathbf{W}$. However, one gains more flexibility in working directly with the posterior distribution of $P$ or $\mu$ given $\mathbf{W}$. That is, $\tilde{f}$, is then seen as one of many possibly interesting functionals of $P$. Moreover, under certain identifiability assumptions, the estimation of the mixing distribution $P$ is of primary concern. 
Notice that the above description shows that the joint distribution of $\left(\mathbf{W}, \mathbf{X}, P, \mathbf{U}_{n}\right)$ can be written as,

$$
\left[\prod_{i=1}^{n} f\left(W_{i} \mid X_{i}\right)\right] \mathbb{P}(d P \mid \mathbf{X}) \mathscr{M}(d \mathbf{X} \mid u) f_{U_{n}}(u)
$$

where $\mathbb{P}(d P \mid \mathbf{X})$ denotes the posterior distribution of $P$ described by Theorem 2.1 or Proposition 2.2. One could then apply arguments similar to those exploited in Lo (1984) and in Ishwaran and James (2003a) to yield analogous characterizations of the posterior distribution. We shall not present those here. In the next section we will describe a general Monte-Carlo which can be used to sample from the posterior distribution. For a better understanding of this connection we note that an application of Proposition 2.4 shows that the marginal distribution of $\mathbf{W}$ is given by

$$
\int_{0}^{\infty}\left[\sum_{\mathbf{p}} \pi_{n}(\mathbf{p} \mid u) \prod_{j=1}^{n(\mathbf{p})} \int_{\mathscr{X}}\left[\prod_{i \in C_{j}} f\left(W_{i} \mid y\right)\right] H_{j, n}(\mathrm{~d} y \mid u)\right] f_{U_{n}}(u) \mathrm{d} u
$$

This is a special case of (14), with $g_{i}(y)=f\left(W_{i} \mid y\right)$.

REMARK 9. It is important to note that in mixture models both $\mathbf{X}$ and $U_{n}$ are viewed as missing values. Hence it is quite natural to work with the distribution of $\mathbf{X} \mid U_{n}$ as a prior and subsequently $\mathbf{X} \mid U_{n}, \mathbf{W}$

\section{Sampling from $\mathscr{M}$ and related functionals}

As mentioned earlier, obtaining a tractable form of the marginal distribution $\mathscr{M}$ is crucial to both practical implementation and theoretical understanding of these models. In particular, understanding how to sample $X_{1}, \ldots, X_{n}$ from $\mathscr{M}$ is important for applications involving mixture models. We discuss briefly some ideas on how this may be done. One aspect of our expressions is the appearance of the cumulants $\kappa_{l}(u)$ and the corresponding moments,

$$
m_{n}(u)=E\left[T_{n(\mathbf{p})}^{n} \mid U_{n}=u\right]
$$

In many cases either the cumulants are easy to calculate or the moments are. Moreover, one can use the following result of Theile in order to recover one from the other. That is, for any integer $n$

$$
\kappa_{n}(u)=m_{n}(u)-\sum_{l=1}^{n-1}\left(\begin{array}{c}
n-1 \\
l-1
\end{array}\right) \kappa_{l}(u) m_{n-l}(u)
$$

Many mathematical packages can easily deal with (16). Similar to the case of the Dirichlet process, it is noted that many complex expressions can be approximated by obtaining draws from $\mathscr{M}$. Using Proposition 2 and Corollary 1 a draw from $\mathscr{M}$ may be conducted as follows. First one draws $U_{n}=\Gamma_{n} / T$, either directly or by drawing from the independent pair $\left(\Gamma_{n}, T\right)$ according to the gamma density of $\Gamma_{n}$ and the density $f_{T}$. Given $U_{n}=u$, one draws $\mathbf{p}$ from $\pi_{n}(\mathbf{p} \mid u)$. Given $\mathbf{p}$ and $U_{n}=u$, one finally draws $\mathbf{X}$ which amounts to independently sampling $Y_{j}$ from $H_{j, n}(\cdot \mid u)$ ), for $j=1, \ldots, n(\mathbf{p})$. Since the normalizing constant, $m_{n}(u)$, in $\pi(\mathbf{p} \mid u)$ is fairly simple, one may often be able to devise a simple scheme to draw $\mathbf{p}$, given $U_{n}=u$, exactly. If this is not the case, one can use a simple variation of a weighted Chinese restaurant (WCR) process [see Lo, Brunner, and Chan (1996) and Ishwaran and James (2003a)], which can be deduced from James (2002, Lemma 2.3). 


\subsection{Generalized Chinese Restaurant and Pólya Urn procedures}

Here, we use the fact that these models are structurally similar to those discussed in section 4 of James (2005a). It follows that one can use section 4.4. of James (2005a) to deduce general extensions of Pólya Urn Gibbs samplers and SIS procedures given by Escobar(1994), Liu (1996), and the Gibbs sampling/SIS procedures based on a generalized Chinese restaurant process as mentioned above. As such, we will only sketch out the relevant probabilities and refer the reader to James (2005a), and references therein, for additional mechanics of the implementation.

For greatest flexibility we will give the relevant probabilities needed to sample approximately from models related to a joint density proportional to

$$
\left[\prod_{i=1}^{n} g_{i}\left(X_{i}\right)\right] \mathscr{M}(d \mathbf{X} \mid u)
$$

which is deducible from Proposition 3.5. We note that $\mathscr{M}(d \mathbf{X} \mid u)$ has an urn type representation which can be deduced from an application of James (2005a, Proposition 5.1). Similar to James (2005a, equation 40), define for $r=0, \ldots, n-1$ conditional probabilities,

$$
\mathbb{P}\left(X_{r+1} \in d x \mid \mathbf{X}_{r}, u\right)=\frac{l_{0, r}}{c_{r}} \lambda_{r}(d y \mid u)+\sum_{j=1}^{n\left(\mathbf{p}_{r}\right)} \frac{l_{j, r}\left(Y_{j}\right)}{c_{r}} \delta_{Y_{j}}(d x)
$$

where $\mathbf{X}_{r}=\left\{X_{1}, \ldots, X_{r}\right\}, \lambda_{r}(d x \mid u) \propto g_{r+1}(x) H_{1,1}(d x \mid u)$,

$$
l_{0, r}=\int_{\mathscr{X}} g_{r+1}(y) H_{1,1}(\mathrm{~d} y \mid u) \quad \text { and } \quad l_{j, r}(y)=g_{r+1}(y) \tau_{1+e_{j, r}}(u \mid y) / \tau_{e_{j, r}}(u \mid y) .
$$

Additionally $c_{r}=l_{0, r}+\sum_{j=1}^{n\left(\mathbf{p}_{r}\right)} l_{j, r}\left(Y_{j}\right)$. Examining James (2005a, section 4.4.) we see these are the ingredients to implement general analogues of the Pólya Urn Gibbs Sampler and SIS procedures described by Escobar (1994) and Liu (1996). To get the Chinese restaurant type procedures one samples partitions $\mathbf{p}$ based on probabilities derived from $l_{0, r}$ and

$$
l_{j, r}=\int_{\mathscr{X}} l_{j, r}(y) \prod_{i \in C_{j, r}} g_{i}(y) H_{j, r}(\mathrm{~d} y \mid u) \text { for } j=1, \ldots, n\left(\mathbf{p}_{r}\right)
$$

where $\mathbf{p}_{r}$ denotes a partion of the integers $\{1, \ldots, r\}$ and each $C_{j, r}=\left\{i \leq r: X_{i}=Y_{j}\right\}$ denotes the corresponding cells. Additionally, $l(r)=l_{0, r}+\sum_{j=1}^{n\left(\mathbf{p}_{r}\right)} l_{j, r}$. In particular, applying the SIS WCR procedure described in James (2005a), now leads to a sampling $\mathbf{p}$ from a density $q(\mathbf{p} \mid u)$, which satisfies

$$
L(\mathbf{p} \mid u) q(\mathbf{p} \mid u)=\pi(\mathbf{p} \mid u) \prod_{j=1}^{n(\mathbf{p})} \int_{\mathscr{X}} \prod_{i \in C_{j}} g_{i}(y) H_{j: n}(d y \mid u)
$$

where $L(\mathbf{p} \mid u)=\prod_{r=1}^{n} l(r-1) / m_{n}(u)$. This is justified by James (2002, Lemma 2.3). Note that setting $g_{i}\left(X_{i}\right)=f\left(W_{i} \mid X_{i}\right)$ leads to sampling procedures for mixture models. Setting $g_{i}(x)=1$ leads to sampling from $\mathscr{M}$. In particular this algorithm includes the classical Chinese restaurant process for the Dirichlet process.

\section{$6 \quad$ Illustrative Examples}

Here we study two examples which are connected to the Dirichlet process, but require a more delicate analysis. In section 7 , we address a more involved class of models. Hereafter, we let $\mathscr{B}(a, b)$, denote the fact that a random variable has a Beta distribution with parameters $(a, b)$. Let $\mathscr{B}(x \mid a, b)$ denote its density. Similarly $\mathscr{G}(a)$ denotes the law of a gamma random variable with shape $a$ and scale 1 . $G_{a}$ denotes the corresponding gamma random variable having density $f_{G_{a}}(x)=\mathscr{G}(x \mid a)$. 


\subsection{Classes of Dependent Dirichlet processes}

Here we present a large class of models which share the same EPPF as the Dirichlet process but are otherwise substantially more complex. This class is seen to add more flexibility to the Dirichlet process, and may be of particular interest in mixture models. One can also see that a study of a subclass of such models is also related to the exposition in Aldous and Pitman (2002).

That is we build NRM based on

$$
\nu_{0, \theta}(d s, d x)=F_{0}(d x \mid s) \theta s^{-1} \mathrm{e}^{-s} .
$$

It is evident that $T \stackrel{d}{=} G_{\theta}$. A careful examination of (17) shows that a class of dependent Dirichlet processes may be described in terms of a stick-breaking representation,

$$
P_{\theta}(\cdot)=\sum_{i=1}^{\infty} \tilde{Q}_{i} \delta_{Z_{i}}(\cdot) \quad \text { with } \quad \tilde{Q}_{i}=W_{i} \prod_{j=1}^{i-1}\left(1-W_{j}\right) .
$$

The $W_{i}$ are independent $\operatorname{Beta}(1, \theta)$, corresponding to the usual representation of Sethuraman (1994), but now the $\left(Z_{i}\right)$ are no longer independent of the $\left(W_{i}\right)$. A technical point is that $\tilde{Q}_{i}=W_{i} \prod_{j=1}^{i-1}(1-$ $\left.W_{j}\right)$ are the points ranked by size-biased sampling and the corresponding $Z_{i}$ has conditional distribution depending on $T \tilde{Q}_{i}=\tilde{J}_{i}$, i.e. $\mathbb{P}\left(Z_{i} \in d z \mid \tilde{J}_{1}, \tilde{J}_{2}, \ldots\right)=F_{0}\left(d z \mid \tilde{J}_{i}\right)$. Note importantly that the distribution of $\tilde{J}_{i}$ is much more manageable than the distribution of the ranked points of a gamma process.

We can describe the posterior distribution in the following way. First, notice that

$$
\nu_{u}(d s, d x)=\mathrm{e}^{-u s} F_{0}(d x \mid s) \theta s^{-1} \mathrm{e}^{-s},
$$

which implies that $\left(1+U_{n}\right) T_{n(\mathbf{p})} \stackrel{d}{=} G_{\theta}$ and $\left(1+U_{n}\right) \mu_{n(\mathbf{p})}(d x) \stackrel{d}{=} G_{\theta} \sum_{i=1}^{\infty} \tilde{Q}_{i} \delta_{Z_{i, n}}$. Here $\left(\tilde{Q}_{i}\right)$ have the same distribution as described above in (18), they are independent of $\mathbf{X}$ and $U_{n}$, while $\left(Z_{i, n}\right)$ are now random variables depending on $U_{n}$. Specifically, conditioned on the sequence $\left(U_{n}, G_{\theta} \tilde{Q}_{1}, G_{\theta} \tilde{Q}_{2}, \ldots\right)$, the $\left(Z_{i, n}\right)$ are independent with distributions

$$
\mathbb{P}\left(Z_{i, n} \in d x \mid U_{n}, G_{\theta} \tilde{Q}_{1}, G_{\theta} \tilde{Q}_{2}, \ldots\right)=F_{0}\left(d x \mid G_{\theta} \tilde{Q}_{i} /\left(1+U_{n}\right)\right)
$$

where $G_{\theta}, U_{n}$ and $\left(\tilde{Q}_{i}\right)$ are independent. Now setting $\left(1+U_{n}\right) J_{j, n}=G_{j, n}$ and one has the conditional distributions of $G_{j, n} \mid U_{n}, \mathbf{X}$ and $G_{j, n} \mid U_{n}, \mathbf{p}$ as,

$$
\mathbb{P}\left(G_{j, n} \in d s \mid Y_{j}, u\right) \propto F_{0}\left(d Y_{j} \mid s /(1+u)\right) \mathscr{G}\left(s \mid e_{j, n}\right) d s
$$

and

$$
\mathbb{P}\left(G_{j, n} \in d s \mid u\right)=\mathscr{G}\left(s \mid e_{j, n}\right) d s .
$$

Additionally, set $T_{n}=\left(1+U_{n}\right) T$. That is $T_{n} \stackrel{d}{=} G_{\theta}+\sum_{j=1}^{n(\mathbf{p})} G_{j, n}$. The conditional density of $V_{n}:=1 /\left(1+U_{n}\right)$, given $\mathbf{X}$ is specified by

$$
f_{V_{n}}(v \mid \mathbf{X}) \propto v^{\theta-1}(1-v)^{n-1} \prod_{j=1}^{n(\mathbf{p})} \int_{0}^{\infty} F_{0}\left(d Y_{j} \mid s v\right) \mathscr{G}\left(s \mid e_{j, n}\right) d s .
$$

$V_{n} \mid \mathbf{p}$ is a $\mathscr{B}(\theta, n)$ random variable, independent of $\mathbf{p}$. We now summarize some facts about this process in the next result.

Theorem 6.1 Suppose that $P_{\theta}$ denotes a class of dependent Dirichlet processes defined as in (18) via the intensity (17). Note that $V_{n}:=1 /\left(1+U_{n}\right)$ given $\mathbf{X}$ has distribution (22). Then the following results hold 
(i) The posterior distribution of $P_{\theta} \mid V_{n}, \mathbf{X}$ is equivalent to the random probability measure

$$
\frac{G_{\theta}}{T_{n}} \sum_{i=1}^{\infty} \tilde{Q}_{i} \delta_{Z_{i, n}}+\sum_{j=1}^{n(\mathbf{p})} Q_{j, n} \delta_{Y_{j}}
$$

where the $\left(\tilde{Q}_{i}\right)$ are equivalent in distribution to those in (18), and are independent of $\mathbf{X}$. The sequence $\left(Z_{i, n}\right)$ has distribution specified by (20). Additionally $Q_{j, n}=G_{j, n} / T_{n}$, where the distribution of $G_{j, n}, T_{n} \mid V_{n}, \mathbf{X}$ is specified by (19) and (21).

(ii) The distributions of $\left(\left(G_{j, n}\right), T_{n},\left(Q_{j, n}\right)\right) \mid \mathbf{p}$, are the same as given $V_{n}$ and $\mathbf{p}$, and equate to the classical results for the Dirichlet process posterior distribution. That is, given $\mathbf{p},\left(Q_{1, n}, \ldots, Q_{n(\mathbf{p}), n}\right)$ is a Dirichlet $\left(e_{1}, \ldots, e_{n(\mathbf{p})} ; \theta\right)$ vector. Equivalently, each $Q_{j, n}$ is $\mathscr{B}\left(e_{j, n}, \theta+n-e_{j, n}\right)$, since the $\left(G_{j, n}\right)$ given $\mathbf{p}$ are independent $\mathscr{G}\left(e_{j, n}, 1\right)$ and $T_{n} \mid \mathbf{p}$ is $\mathscr{G}(\theta+n)$.

It is evident again that the prediction rule does not in general have a simple form. However, the next result yields a nice description of the marginal distribution of $\mathbf{X}$.

Proposition 6.1 Suppose that $P_{\theta}$ is a dependent Dirichlet process as described above. Then the marginal distribution of $\mathbf{X} \mid V_{n}=v$, where $V_{n}$ is $\mathscr{B}(\theta, n)$, is given by

$$
P D(\mathbf{p} \mid \theta) \prod_{j=1}^{n(\mathbf{p})} \int_{0}^{\infty} F_{0}\left(d y_{j} \mid s v\right) \mathscr{G}\left(s \mid e_{j, n}\right) d s
$$

The expression in (23) shows that the conditional distribution of $Y_{1}, \ldots, Y_{n(\mathbf{p})} \mid V_{n}=v, \mathbf{p}$ are independent with respective distributions

$$
\mathbb{P}\left(Y_{j} \in d y \mid v\right)=\int_{0}^{\infty} F_{0}\left(d y_{j} \mid s v\right) \mathscr{G}\left(s \mid e_{j, n}\right) d s .
$$

Additionally, $Y_{1}, \ldots, Y_{n(\mathbf{p})} \mid\left(G_{j, n}\right), V_{n}=v, \mathbf{p}$ are independent with distributions $F_{0}\left(d y_{j} \mid G_{j, n} v\right)$.

Notice that in every case the distribution of $\left(Y_{j}\right) \mid V_{n}, \mathbf{p}$ are expressible via gamma mixing measures. The next result demonstrates a particularly simple case.

Proposition 6.2 Suppose that for $a>0, \delta>0, F_{0}(d y \mid a) \propto a^{\delta} y^{\delta-1} e^{-a y} d y$ corresponds to a gamma random variable. Then $Y_{1}, \ldots, Y_{n(\mathbf{p})} \mid V_{n}, \mathbf{p}$ are independent with each $Y_{j} \stackrel{d}{=} R_{j} / V_{n}$, where $R_{j}$ is independent of $V_{n}$ and the distribution of $R_{j} \mid \mathbf{p}$ is

$$
f_{R_{j}}(r \mid \mathbf{p}) \propto r^{\delta-1}(1+r)^{-\left(\delta+e_{j, n}\right)} \text { for } 0<r<\infty
$$

Or equivalently $1 /\left(1+R_{j}\right)$ given $\mathbf{p}$ is $\mathscr{B}\left(e_{j, n}, \delta\right)$. Additionally the $Z_{i, n}$ appearing in Proposition 6.1 satisfy $Z_{i, n} \stackrel{d}{=} \tilde{R}_{i} /\left(\tilde{Q}_{i} V_{n}\right)$ where $1 /\left(1+\tilde{R}_{i}\right)$ is independent of $\left(\tilde{Q}_{i}\right), V_{n}$ and has a $\mathscr{B}(1, \delta)$ distribution. The distribution of the $Z_{i}$ is given by setting $V_{0}:=1$

REMARK 10. Note that unlike the case of the usual Dirichlet process $V_{n}$ is not independent of $\mathbf{X}$, however its distribution is independent of $\mathbf{p}$ and the marginal distribution of $V_{n}$ is the same in both the dependent and classical case. Proposition 6.2 shows that it is easy to sample from $\mathscr{M}$. Since $V_{n}, \mathbf{p}$ are independent, one can draw from $P D(\mathbf{p} \mid \theta)$ according to the classical Chinese restaurant, then sample independently $V_{n}$ from a $\mathscr{B}(\theta, n)$. It then remains to draw the $Y_{1}, \ldots, Y_{n(\mathbf{p})}$ conditional on $V_{n}, \mathbf{p}$. Or as an intermediate step, conditional on $\left(G_{j, n}\right), V_{n}=v, \mathbf{p}$. 


\subsection{NRMs derived from Beta processes}

Suppose that $\rho(\mathrm{d} s \mid x)=s^{-1}(1-s)^{c(x)-1} d s c(x)$, for $0<s \leq 1$. The corresponding process $\mu$ is a beta process. Exploiting beta processes as an ingredient for constructing NRM models leads to a different posterior behaviour from the one analyzed in Hjort (1990). The Lévy measure associated with $\mu_{u}$ in (5) is

$$
\nu_{u}(\mathrm{~d} s, \mathrm{~d} x)=\mathrm{e}^{-u s} s^{-1}(1-s)^{c(x)-1} \mathrm{~d} s \eta(\mathrm{d} x) \quad \text { for } \quad s \in(0,1]
$$

and, hence, $\mu_{u}$ is not a beta process. Additionally, the distribution of the jumps $J_{j, n}$, given $\left(U_{n}, \mathbf{X}\right)$ is

$$
\mathbb{P}\left(J_{j, n} \in \mathrm{d} s \mid Y_{j}, u\right)=\frac{1}{{ }_{1} F_{1}\left(e_{j, n}, c\left(y_{j}\right)+e_{j, n} ;-u\right)} \mathrm{e}^{-u s} s^{e_{j, n}-1}(1-s)^{c\left(y_{j}\right)-1} \mathrm{~d} s
$$

where ${ }_{1} F_{1}$ is the confluent hypergeometric function. It follows that one can write $m\left(\mathrm{~d} x_{i} \mid x_{1}, \ldots, x_{i-1}, u\right)$ as,

$$
\begin{aligned}
&{ }_{1} F_{1}\left(1, c\left(x_{i}\right)+1 ;-u\right) \eta\left(\mathrm{d} x_{i}\right)+ \\
& \sum_{j=1}^{n\left(\mathbf{p}_{i-1}\right)} \frac{e_{j, i-1}}{e_{j, i-1}+c\left(y_{j}\right)} \frac{{ }_{1} F_{1}\left(e_{j, i-1}+1, c\left(y_{j}\right)+e_{j, i-1}+1 ;-u\right)}{{ }_{1} F_{1}\left(e_{j, i-1}, c\left(y_{j}\right)+e_{j, i-1} ;-u\right)} \delta_{y_{j}}\left(\mathrm{~d} x_{i}\right) .
\end{aligned}
$$

Finally, the marginal distribution of $\mathbf{X}$, given $U_{n}$, can be represented as

$$
\left[m_{n}(u)\right]^{-1}\left[\prod_{j=1}^{n(\mathbf{p})} \frac{\Gamma\left(e_{j, n}\right) \Gamma\left(c\left(y_{j}\right)+1\right)}{\Gamma\left(e_{j, n}+c\left(y_{j}\right)\right)}\right] \prod_{j=1}^{n(\mathbf{p})}{ }_{1} F_{1}\left(e_{j, n}, c\left(y_{j}\right)+e_{j} ;-u\right) \eta\left(\mathrm{d} y_{j}\right)
$$

where $m_{n}(u)$ can be expressed using (16), where $\kappa_{1}(u)=m_{1}(u)$.

REMARK 11. We point that by setting $c(s):=1$ and letting $\eta(d x)=\theta H(d x)$, yields models defined by the scale invariant Poisson process, which is of importance in a variety of applications. In particular it is known that $T$ has the important Dickman distribution. Moreover, in this case, the class of NRM have been discussed previously. Specifically, Arratia, Barbour and Tavare (1999, 2004) show that the distribution of $P \mid T \leq 1$ is in fact a Dirichlet process with shape $\theta$. See Arratia, Barbour and Tavare (2004) for further implications and details.

\section{Generalized Gamma Convolution processes: NRM related to Dirichlet mean functionals}

This last example demonstrates the great flexibility of the $h$-biased framework, which allows us to describe a large class of NRM in terms of the tractable $\left(\tilde{Q}_{i}\right)$ of the Dirichlet process and related variables. These models will be based on random variables $T$ which have distributions that are $G e n-$ eralized Gamma Convolutions (GGC). This large class of self-decomposable infinite-divisible random variables was introduced by Thorin $(1977,1978)$, further developments are given in Bondesson (1979, 1992). In particular, a subclass of such models will be seen to be connected to the study of mean functionals of a Dirichlet process, initiated by Cifarelli and Regazzini (1990). We believe that our discussion will also shed some new light on these two lines of research which are essentially duals of one another. Moreover, our approach shows that one may extend the study of mean functionals of Dirichlet processes to this larger and more flexible setting. Quite strikingly, this class of NRM is very rich, including the stable law processes of index $0<\alpha<1$, (and hence by a change of variable the entire two parameter Poisson Dirichlet class discussed in Pitman (1996)), classes of models based 
on the Pareto and log-Normal distribution, and the class of Generalized Inverse Gaussian (GIG) models, among many others.

Let $N$ denote a Poisson random measure on the space $(0, \infty) \times(0, \infty) \times \mathscr{X}$, with mean intensity

$$
\nu(d r, d v, d x)=\theta r^{-1} \mathrm{e}^{-r} \mathscr{U}(d v) H(d x)
$$

where $\mathscr{U}$ is a non-negative non-decreasing function on satisfying, $\mathscr{U}(0)=0$,

$$
\int_{0}^{1}|\ln (v)| \mathscr{U}(d v)<\infty \text { and } \int_{1}^{\infty} 1 / y \mathscr{U}(d y)<\infty .
$$

Note importantly, that it is possible for $\mathscr{U}(\infty)=\infty$. In particular, the condition (25) is true if and only if

$$
\psi(\lambda)=\theta \int_{0}^{\infty} \int_{0}^{\infty}\left(1-\mathrm{e}^{-\lambda(r / v)}\right) r^{-1} \mathrm{e}^{-r} \mathscr{U}(d v)=\theta \int_{0}^{\infty} \ln (1+\lambda / v) \mathscr{U}(d v)<\infty
$$

This allows one to define random variables $T=G_{\theta} M_{\theta}=\int_{0}^{\infty} \int_{0}^{\infty}(r / v) N(d r, d v)$, where $G_{\theta}$ is independent of $M_{\theta}$, and has Lévy exponent $\psi(\lambda)$. Let $\mathscr{T}$ denote the family of all random variables, $T$, such that the Lévy exponent of $T$ satisfies (26). That is $\mathscr{T}$ is the class of random variables with distributions which are generalized gamma convolutions. This may be extended by random variables $T+a$ for $a \geq 0$. The quantity $\mathscr{U}$ uniquely determines the distribution of $T$, and we refer to $\mathscr{U}$ as the Thorin measure. Additionally,

$$
\mu(d x)=G_{\theta} \sum_{i=1}^{\infty}\left(\tilde{Q}_{i} / V_{i}\right) \delta_{Z_{i}}(d x)=\int_{0}^{\infty} \int_{0}^{\infty}(r / v) N(d r, d v, d x)
$$

and $M_{\theta}=\sum_{i=1}^{\infty}\left(\tilde{Q}_{i} / V_{i}\right) . \mu$ may be referred to as a GGC random measure. Then call $P_{M_{\theta}}$ a GGC NRM if it has a representation as an $h$-biased random probabilty measure, here $s:=(r, v)$, with $h(r, v)=r / v$, given by

$$
P_{M_{\theta}}(d x)=\frac{\sum_{i=1}^{\infty}\left(\tilde{Q}_{i} / V_{i}\right) \delta_{Z_{i}}(d x)}{M_{\theta}}=\frac{\int_{0}^{\infty} \int_{0}^{\infty}(r / v) N(d r, d v, d x)}{T}
$$

where $\left(\tilde{Q}_{i}\right)_{i}$ has the same marginal distribution as in (18), but is now independent of the sequence $\left(Z_{i}\right)_{i}$ of i.i.d. random variables whose distribution is $H$. Additionally, both sequences are independent of $\left(V_{i}\right)_{i}$. Note that the distribution of the sequence is derived from the points of a Poisson random measure with mean intensity $\mathscr{U}$. In the special case where $\mathscr{U}$ is a probability measure (finite measure), the $\left(V_{i}\right)_{i}$ are i.i.d. $\mathscr{U}$. However this not always true. In fact the obtainment of many interesting classes, such as the stable law, require that $\mathscr{U}$ is not a finite measure.

REMARK 12. We mention that if $\mathscr{U}$ is a finite measure then $M_{\theta} \stackrel{d}{=} \int_{0}^{\infty} 1 / y D_{\theta}(d y), D_{\theta}$ being a Dirichlet process with shape parameter $\theta \mathscr{U}$. That is $M_{\theta}$ corresponds to a class of (positive) Dirichlet mean functionals. That is, a subset of the Dirichlet process functionals whose study was initiated by Regazzini and Cifarelli (1990). However $M_{\theta}$ constitutes a wider class of positive random variables as the representation $\sum_{i=1}^{\infty} \tilde{Q}_{i} \delta_{V_{i}, Z_{i}}$ does not in general correspond to a Dirichlet process unless the $V_{i}$ 's are iid. Rather $G_{\theta} \sum_{i=1}^{\infty} \tilde{Q}_{i} \delta_{V_{i}, Z_{i}}$ is a gamma process with possibly sigma-finite shape measure $\theta \mathscr{U} H$. As we shall see, this generality of $M_{\theta}$ allows us much greater flexibility as there are many cases where the distribution of $M_{\theta}$ and $T$ can be deduced.

REMARK 13. Note interestingly, by independence of $G_{\theta}$ and $M_{\theta}$, the fact that $G_{\theta}$ is gamma distributed yields,

$$
E\left[\mathrm{e}^{-u T}\right]=E\left[\mathrm{e}^{-u G_{\theta} M_{\theta}}\right]=\int_{0}^{\infty}(1+u v)^{-\theta} f_{M_{\theta}}(v) d v=\mathrm{e}^{-\theta \int_{0}^{\infty} \ln (1+u / y) \mathscr{U}(d y)}
$$


where $f_{M_{\theta}}$ denotes the density of $M_{\theta}$. This is essentially the identity established in Cifarelli and Regazzini (1990) for Dirichlet mean functionals. Note that the choice of $\mathscr{U}$, uniquely determines the distribution of $M_{\theta}$. Hence when $\mathscr{U}(\infty)=\infty$, such distributions are not captured by the current literature on Dirichlet mean functionals. Note additionally that the theory of GGC has been extended by Thorin (1978) [see Bondesson (1992)] to include distributions on the entire real line. See also Lijoi and Regazzini (2004). Here of course we require that $T$ is positive.

\subsection{Posterior Distribution of GGC NRM}

Now to establish the posterior distribution, first note that $\mu_{n(\mathbf{p})} \mid U_{n}=u, \mathbf{X}$ has mean intensity

$$
\nu_{u}(d r, d v, d x)=\theta r^{-1} \mathrm{e}^{-r(1+u / v)} \mathscr{U}(d v) H(d x)
$$

We recognize, from James (2005a, Proposition 2.1), that the change in the intensity from $\nu$ to $\nu_{u}$ is due to exponential tilting by $\mathrm{e}^{-u T}$. It is useful to see explicitly how this operation affects the Thorin measure, $\mathscr{U}$, and indeed how this affects the resulting distribution of $M_{\theta}$.

Proposition 7.1 Let $T \in \mathscr{T}$ defined by the Thorin measure $\mathscr{U}(d v):=\omega(v) d v$. Then suppose that $T_{b}$ is the random variable with density $\propto e^{-b t} f_{T}(t)$ for some $b>0$. Then it follows that $T_{b} \in \mathscr{T}$, with Thorin measure $\omega(z-b) I\{z>b\} d z$. This follows from the fact that its Lévy exponent is expressible as

$$
\psi_{b}(\lambda)=\int_{b}^{\infty} \ln (1+\lambda / z) \omega(z-b) d z=\int_{0}^{\infty} \ln (1+\lambda /(y+b)) \omega(y) d y .
$$

Equivalently, $T_{b} \stackrel{d}{=} G_{\theta} M_{\theta, b}=\int_{0}^{\infty} \int_{0}^{\infty}(r / y) N_{b}(d r, d y)$, where $N_{b}$ is a Poisson random measure with $\mathbb{E}\left[N_{b}(d r, d z, d x)\right]=\theta r^{-1} e^{-r} \omega(z-b) I\{z>b\} d z H(x) . M_{\theta, b}:=\sum_{i=1}^{\infty} \tilde{Q}_{i} /\left(V_{i}+b\right)$ is the mean functional whose law is induced by the tilting operation

Proof. This follows from a straightforward change of variables or using the fact that $\psi_{b}(\lambda)=$ $\psi(\lambda+b)-\psi(b)$.

Now examining Proposition 7.1, with $b=U_{n}$, it follows that,

$$
\mu_{n(\mathbf{p})} \stackrel{d}{=} G_{\theta} \sum_{i=1}^{\infty} \tilde{Q}_{i}\left(V_{i}+U_{n}\right)^{-1} \delta_{Z_{i}}
$$

Additionally $T_{n(\mathbf{p})} \stackrel{d}{=} G_{\theta} M_{\theta, U_{n}}$. Setting $J_{j, n}:=\left(\Delta_{j, n}, V_{j, n}\right)$, its joint distribution given $U_{n}, \mathbf{X}$ is given by,

$$
\mathbb{P}\left(\Delta_{j, n} \in d r, V_{j, n} \in d v \mid u\right)=\frac{\mathrm{e}^{-r(1+u / v)} r^{e_{j}-1} v^{-e_{j}} \theta \mathscr{U}(d v) d r}{\kappa_{e_{j}}(u)},
$$

where $\kappa_{e_{j}}(u)=\theta \Gamma\left(e_{j, n}\right) \int_{0}^{\infty}(v+u)^{-e_{j}} \mathscr{U}(d v)$. This shows that the distribution of each $\Delta_{j, n} \mid V_{j, n}, U_{n}$ is equivalent in distribution to $G_{j, n}\left(1+U_{n} / V_{j, n}\right)^{-1}$, where $G_{j, n}$ denotes a gamma random variable with shape $e_{j}$ and scale 1 independent of $\left(U_{n}, V_{j, n}\right)$. The distribution of $V_{j, n} \mid U_{n}, \mathbf{X}$ is given by the density

$$
\mathbb{P}\left(V_{j, n} \in d v \mid u\right)=\frac{(v+u)^{-e_{j}} \theta \Gamma\left(e_{j}\right) \mathscr{U}(d v)}{\kappa_{e_{j}}(u)}=\frac{(v+u)^{-e_{j}} \mathscr{U}(d v)}{\int_{0}^{\infty}(y+u)^{-e_{j}} \mathscr{U}(d y)}
$$

Additionally, we use the fact that the prior and posterior distribution of

$$
U_{n} T=U_{n} G_{\theta} M_{\theta}=U_{n}\left[G_{\theta} \sum_{i=1}^{\infty} \tilde{Q}_{i}\left(V_{i}+U_{n}\right)^{-1}+\sum_{j=1}^{n(\mathbf{p})} G_{j, n}\left(V_{j, n}+U_{n}\right)^{-1}\right] \stackrel{d}{=} \Gamma_{n},
$$


where $\Gamma_{n}$ is independent of $N$ and $\mathbf{X}$. Furthermore notice that,

$$
m_{n}(u)=E\left[T_{n(\mathbf{p})}^{n} \mid u\right]=\frac{\Gamma(\theta+n)}{\Gamma(\theta)} \frac{\int_{0}^{\infty}(1+u v)^{-(\theta+n)} v^{n} f_{M_{\theta}}(d v)}{\int_{0}^{\infty}(1+u v)^{-\theta} f_{M_{\theta}}(d v)}
$$

These facts lead to a non-obvious description of the posterior distribution given $U_{n}, \mathbf{X}$.

Theorem 7.1 Suppose that $P_{M_{\theta}}$, is the NRM defined by (27). Then the following results hold

(i) The posterior distribution of $P_{M_{\theta}} \mid U_{n}, \mathbf{X}$ is equivalent to the distribution of the random probability measure

$$
U_{n} \frac{G_{\theta+n}}{\Gamma_{n}}\left[\frac{G_{\theta}}{G_{\theta+n}} \sum_{i=1}^{\infty} \tilde{Q}_{i}\left(V_{i}+U_{n}\right)^{-1} \delta_{Z_{i}}+\sum_{j=1}^{n(\mathbf{p})} Q_{j, n}\left(V_{j, n}+U_{n}\right)^{-1} \delta_{Y_{j}}\right]
$$

where, the gamma random variable $G_{\theta+n}=G_{\theta}+\sum_{j=1}^{n(\mathbf{p})} G_{j, n}$, and independent of $U_{n}, \Gamma_{n},\left(Z_{i}\right)$, the $\left(\tilde{Q}_{i}\right)$ are equivalent in distribution to those in [18), and are independent of $\mathbf{X}$. Additionally, the vector $\left(Q_{1, n}, \ldots, Q_{n(\mathbf{p}), n}\right)$ is independent of $\left(U_{n}, \Gamma_{n},\left(Z_{i}\right), G_{\theta}, G_{\theta+n}\right)$ and given $\mathbf{p}$, is a Dirichlet $\left(e_{1}, \ldots, e_{n(\mathbf{p})} ; \theta\right)$ vector. The relevant distributions of $V_{j, n}, U_{n}$ are specified by (28) and (32).

(ii) Equivalently, one may write (30) as

$$
\frac{U_{n} T_{n(\mathbf{p})}}{\Gamma_{n}} \sum_{i=1}^{\infty} \tilde{Q}_{i, n} \delta_{Z_{i}}+\left[1-\frac{U_{n} T_{n(\mathbf{p})}}{\Gamma_{n}}\right] \sum_{j=1}^{n(\mathbf{p})} Q_{j, n}^{*} \delta_{Y_{j}}
$$

where

$$
\tilde{Q}_{i, n}=\frac{\tilde{Q}_{i}\left(V_{i}+U_{n}\right)^{-1}}{\sum_{i=1}^{\infty} \tilde{Q}_{l}\left(V_{l}+U_{n}\right)^{-1}} \quad \text { and } \quad Q_{j, n}^{*}=\frac{Q_{j, n}\left(V_{j, n}+U_{n}\right)^{-1}}{\sum_{l=1}^{n(\mathbf{p})} Q_{l, n}\left(V_{l, n}+U_{n}\right)^{-1}}
$$

\section{2 $\mathscr{M}$ and some connections to the Bondesson Class}

Before saying more about the distribution of $U_{n}, \mathbf{X}$, we next describe an important subclass of GGC random variables which interestingly are connected to the distribution of $U_{n}$. As in Kent and Tyler $(2001$, p.257) let $S$ denote a random variable on $(0, \infty)$ with density

$$
f_{S}(s)=C s^{\beta-1} \prod_{j=1}^{m}\left(1+c_{j} s\right)^{-\gamma_{j}} \text { for } s>0,
$$

where $C$ is a normalizing constant, $m \geq 1, \beta>0, c_{j}>0, \gamma_{j}>0$. Then the class containing the densities (31), together with their weak limits, constitutes the Bondesson $\mathscr{B}$ sub-class of GGC models. We write $S \in \mathscr{B}$ if the density of $S$ has the form in (31). Note that in the non-limiting case, the corresponding $\mathscr{U}(\infty)=\beta<\infty$. That is, in this case, the $\left(V_{i}\right)$ are iid with distribution $\beta^{-1} \mathscr{U}$. The $\mathscr{B}$ class contains the Stable distributions of index $\alpha=1 / k$ for $k=2,3 \ldots$. The gamma distribution, Pareto, Log-Normal random variables, generalized inverse gaussian, among many others. [See Bondesson(1992, Chapter 5.6)]. This class is known to be hyperbolically completely monotone and hence self-decomposable. See Bondesson (1992), Steutel and Van Harn (2004, Chapter $5)$ and Kent and Tyler (2001, p.257) for further details.

Proposition 7.2 Let $U_{n}=\Gamma_{n} / T=\Gamma_{n} /\left(G_{\theta} M_{\theta}\right)$, then its distribution is described as follows. 
(i) The density of $U_{n} \mid \mathbf{p}$ is,

$$
f_{U_{n}}(u \mid \mathbf{p}) \propto u^{n-1}\left[\int_{0}^{\infty}(1+u v)^{-\theta} f_{M_{\theta}}(d v)\right] \prod_{j=1}^{n(\mathbf{p})} \int_{0}^{\infty}(v+u)^{-e_{j}} \mathscr{U}(d v)
$$

(ii) The marginal density of $U_{n}$ is

$$
\frac{u^{n-1}}{\Gamma(n)} \mathbb{E}\left[e^{-u G_{\theta} M_{\theta}}\left(G_{\theta} M_{\theta}\right)^{n}\right]=u^{n-1} \frac{\Gamma(\theta+n)}{\Gamma(n) \Gamma(\theta)} \int_{0}^{\infty}(1+u v)^{-(\theta+n)} v^{n} f_{M_{\theta}}(d v)
$$

This implies that that for any integrable function $\mathrm{g}$,

$$
\mathbb{E}\left[g\left(U_{n}\right)\right]=\int_{0}^{1} \mathbb{E}\left[g\left(\frac{1-y}{y M_{\theta}}\right)\right] \mathscr{B}(d y \mid \theta, n)
$$

Proposition 7.3 Suppose that $T \in \mathscr{T}$, then $U_{n}:=\Gamma_{n} / T=\Gamma_{n} /\left(G_{\theta} M_{\theta}\right)$ has the following properties.

(i) If $T:=G_{\theta} M_{\theta} \in \mathscr{B}$, then $U_{n} \in \mathscr{B}$ However, $T \in \mathscr{T}$ does not imply that $U_{n} \in \mathscr{T}$.

(ii) The distribution of $U_{n} \mid M_{\theta}=v$ is in $\mathscr{B}$. with density of the form in (31), with parameters, $\beta=n, m=1, c_{1}=v, \gamma_{1}=\theta+n$. Equivalently, $M_{\theta} U_{n}$ is a gamma-gamma density, and hence in $\mathscr{B}$. The form of the density coincides with $U_{n} \mid M_{\theta}=1$. That is $c_{1}=1$

(iii) The distribution of $U_{n} \mid M_{\theta}=v,\left(V_{j, n}=v_{j}\right), \mathbf{p}$ is in $\mathscr{B}$, with $\beta=n, m=n(\mathbf{p})+1, c_{j}=1 / v_{j}$, $\gamma_{j}=e_{j}$, for $j=1, \ldots, n(\mathbf{p})$ and $c_{n(\mathbf{p})+1}=v, \gamma_{n(\mathbf{p})+1}=\theta$. Specifically the conditional density is given by

$$
f_{U_{n}}\left(u \mid v,\left(v_{j}\right)\right)=C u^{n-1}(1+u v)^{-\theta} \prod_{j=1}^{n(\mathbf{p})}\left(1+u / v_{j}\right)^{-e_{j}}
$$

(iv) Let $Y_{n}=n / U_{n}$. Then $Y_{n} \in \mathscr{T}$ and hence this family of densities is dense in the class of all $\mathscr{T}$.

Proof. Statement (i) is an immediate consequence of Bondesson (1992, Theorem 6.2.1, p.92.). See also Kent and Tyler (2001, statement 7, p. 257). Statement (ii) and (iii) follow from an augmentation and matching with (31). Statement(iv) is read from Bondesson (1992, p. 92).

The next result gives a form of the EPPF.

Proposition 7.4 Suppose that $\mathbf{p}$ denotes the partition derived from $P_{M_{\theta}}$. Then the following results hold.

(i) The conditional distribution of $\mathbf{p} \mid U_{n}$ is given by

$$
P D(\mathbf{p} \mid \theta)\left[\frac{\int_{0}^{\infty}(1+u v)^{-\theta} f_{M_{\theta}}(d v)}{\int_{0}^{\infty}(1+u v)^{-(\theta+n)} v^{n} f_{M_{\theta}}(d v)}\right] \prod_{j=1}^{n(\mathbf{p})} \int_{0}^{\infty}(y+u)^{-e_{j}} \mathscr{U}(d y)
$$

(ii) The EPPF may be expressed as,

$$
\begin{aligned}
P D(\mathbf{p} \mid \theta) \frac{\Gamma(\theta+n)}{\Gamma(n) \Gamma(\theta)} \int_{0}^{\infty} u^{n-1}\left[\int_{0}^{\infty}(1+u v)^{-\theta} f_{M_{\theta}}(d v)\right] \times & \\
& {\left[\prod_{j=1}^{n(\mathbf{p})} \int_{0}^{\infty}(y+u)^{-e_{j}} \mathscr{U}(d y)\right] d u }
\end{aligned}
$$


REMARK 14. The result in (16) combined with (29) and the form of $\kappa_{j}$ leads to interesting relationships between $\mathscr{U}$ and $M_{\theta}$. In particular, using the property that $\kappa_{1}(u)=m_{1}(u)$ leads to the following identity,

$$
\int_{0}^{\infty}(v+u)^{-1} \mathscr{U}(d v)=\frac{\int_{0}^{\infty}(1+u v)^{-(\theta+1)} v f_{M_{\theta}}(d v)}{\int_{0}^{\infty}(1+u v)^{-\theta} f_{M_{\theta}}(d v)}
$$

REMARK 15. The unified representation of $P_{M_{\theta}}$ and the characterization of its posterior distribution given in (30) in terms of the Dirichlet process $\left(Q_{i}\right)$ has many interesting implications. For instance, it suggests that one can use a variant of the Blocked Gibbs algorithms in Ishwaran and James $(2001,2003 \mathrm{~b})$ to approximately sample realizations of $P_{M_{\theta}}$ and its posterior process for many different classes of models which are not Dirichlet processes.

\subsection{Some specific examples of GGC NRM}

In this section we now highlight some important specific cases. First it is interesting to note the the study of the GGC is primarily about establishing the fact that $T \in \mathscr{T}$, and possibly identifying $\mathscr{U}$. In contrast, the study of Dirichlet process mean functionals involves identification of the distribution of $M_{\theta}$, when $\mathscr{U}$ is a pre-specified finite measure. We see these two approaches as complementary to one another. We point out that explicit forms for the $\mathscr{U}$ are not known in every case. However, importantly there are many examples of $T$ which are known to be in $\mathscr{T}$. For instance, an explicit form of $\mathscr{U}$ is not known for the Pareto distribution. However, as we have shown, many interesting applications involving sampling from $\mathscr{M}$ and mixture models can still be conducted if one knows $m_{n}(u)$ or the cumulants $\kappa_{n}(u)$. Similarly, from the Dirichlet process literature the explicit law of $M_{\theta}$ is not known in many cases, however we can choose $\mathscr{U}$ to be from a vast selection of probability distributions. Thus as stated earlier, in that case, we have an explicit description of the iid distribution of the sequence $\left(V_{i}\right)$.

As some general examples, one could choose $T=S \in \mathscr{B}$, as defined in (31). Note also that $T=S^{q} \in \mathscr{T}$ for $|q| \geq 1$ [see Bondesson (1979, Corollary 1)]. Here we give some precise examples from the literature.

REMARK 16. Note carefully that we only need to show that $T$ has a particular law to establish the law of the NRM $P_{M_{\theta}}:=\mu / T$. This is due to the fact that the Laplace functional of $\mu$ evaluated at some bounded measurable functional $g$ is determined by

$$
-\log \mathbb{E}\left[\mathrm{e}^{-\mu(g)}\right]=\int_{\mathcal{X}} \psi(g(x)) H(d x)
$$

for $\psi(g(x))$ given by replacing $\lambda$ with $g(x)$ in (26)

\subsubsection{Stable Case and related models}

As mentioned previously, the NRM based on the stable law have been extensively studied by Pitman (1996, 2002) and Pitman and Yor (1997). This class has numerous applications and also has a tractable EPPF. The explicit posterior distribution of this class was obtained by Pitman (1996) by exploiting its explicit stick-breaking representation. James (2002, section 5.3) gives an alternative derivation working directly with the Levy measure of a stable law. Here, we show that $P_{M_{\theta}}$ offers another representation of the stable law NRM and hence alternative approach to its analysis. We give some details of its posterior analysis which are inherent to its representation in terms of a GGC NRM. Further details can be deduced easily from the specific analysis of these models in James (2002, sections 5.3 and 5.4).

From Bondesson $(1992$, p. 35$)$, it is now easy to see that $T$ is stable, and hence $P_{M_{\theta}}$ is a stable NRM, if

$$
\mathscr{U}_{\alpha}(d v)=\frac{\alpha}{\Gamma(1-\alpha) \Gamma(\alpha)} v^{\alpha-1} d v \text { for } v>0
$$


As checks, one can make the change of variable $z=r / v$, in (26) and integrate with respect to $\mathscr{U}$ first. That is for all $\theta>0, \psi(\lambda)=C_{\alpha, \theta} \lambda^{\alpha}$ for some constant $C_{\alpha, \theta}$. Note interestingly that, conditional on $U_{n}$, we have

$$
\left(\frac{V_{j, n}}{U_{n}}+1\right)^{-1} \sim \mathscr{B}\left(\mathrm{e}_{j}-\alpha, \alpha\right)
$$

and, hence,

$$
G_{j, n}\left(\frac{V_{j, n}}{U_{n}}+1\right)^{-1} \sim \mathscr{G}\left(e_{j}-\alpha\right)
$$

for $j=1, \ldots, n(\mathbf{p})$. That is, these quantities are independent of $U_{n}$. Additionally, $\kappa_{e_{j}}(u)=$ $\theta \alpha u^{\alpha-e_{j}} \Gamma\left(e_{j}-\alpha\right) / \Gamma(1-\alpha)$. Ignoring scale parameters it follows that the distribution of $L_{n}:=U_{n}^{\alpha} \mid \mathbf{p}$ is $\mathscr{G}(n(\mathbf{p}))$. One then establishes that $\mu_{n(\mathbf{p})} \mid U_{n}, \mathbf{p}$ is a generalized gamma process, whose Thorin measure is given by (38) below, with $b=U_{n}$. Moreover the distribution of $L_{n}^{1 / \alpha} \mu_{n(\mathbf{p})} \mid L_{n}, \mathbf{p}$ is determined by the Lévy measure

$$
\frac{L_{n} \alpha}{\Gamma(1-\alpha) \Gamma(\alpha)} r^{-1} \mathrm{e}^{-r}(v-1)^{\alpha-1} I\{v>1\} d r d v H(d x) .
$$

In particular this implies that the distribution of $L_{n}^{1 / \alpha} T_{n(\mathbf{p})} \mid \mathbf{p}$ is $\mathscr{G}(n(\mathbf{p}) \alpha)$. These formula can be used in Proposition 7.1 and 7.3, to establish the known results about the posterior distribution. Further details can be deduced from James (2002, section 5). The two parameter Poisson-Dirichlet distribution with parameters $(\alpha, q)$ for $0<\alpha<1$ and $q>-\alpha$ arises from the weighted law $\propto T^{-q} \mathbb{P}(d N \mid \nu)$ as described in Proposition 8.2. It remains to note that $L_{n, q}:=U_{n, q}^{\alpha}$ given $\mathbf{p}$ is $\mathscr{G}(n(\mathbf{p})+q \alpha)$

REMARK 17. Note also that, in the case where $T$ is the stable law, the generalized CauchyStieltjes transform of $M_{\theta}$ is

$$
\int_{0}^{\infty}(1+\lambda v)^{-\theta} f_{M_{\theta}}(d v)=\mathrm{e}^{-C_{\alpha, \theta} \lambda^{\alpha}}
$$

which can be easily inverted. This, in some sense, easiest example is outside of the scope of the current theory of Dirichlet process mean functionals as $\mathscr{U}(\infty)=\infty$.

\subsubsection{Generalized Gamma}

The class of generalized gamma process defined for $0<\alpha<1$, and $b>0$ [see Brix (1999)] arises from the tilting by $\mathrm{e}^{-b S_{\alpha}}$, where $S_{\alpha}$ is stable law of index $\alpha$. The simplest case is when $\alpha=1 / 2$, where the corresponding $T$ has an Inverse Gaussian distribution. Proposition 7.1 shows that the Thorin measure is given, in this case, by

$$
\frac{\alpha}{\Gamma(1-\alpha) \Gamma(\alpha)}(v-b)^{\alpha-1} d v \quad \text { for } \quad v>b .
$$

The Thorin measure of $\mu_{n(\mathbf{p})} \mid U_{n}$, $\mathbf{p}$ is of the same form as (38) with $b$ replaced by $U_{n}+b$, and hence is a generalized gamma process. It follows that setting $L_{n}=C_{\alpha, \theta}\left(U_{n}+b\right)^{\alpha}$, the distribution of $L_{n}^{1 / \alpha} \mu_{n(\mathbf{p})} \mid L_{n}, \mathbf{p}$ is the same as that for the stable law determined by (37). Similar to (36) one has, conditional on $U_{n}$,

$$
\left(\frac{V_{j, n}-b}{U_{n}+b}+1\right)^{-1} \sim \mathscr{B}\left(\mathrm{e}_{j}-\alpha, \alpha\right) \quad \text { and } \quad G_{j, n}\left(\frac{V_{j, n}-b}{U_{n}+b}+1\right)^{-1} \sim \mathscr{G}\left(e_{j}-\alpha\right)
$$

for $j=1, \ldots, n(\mathbf{p})$. With $\kappa_{e_{j}}(u)=\theta \alpha(u+b)^{\alpha-e_{j}} \Gamma\left(e_{j}-\alpha\right) / \Gamma(1-\alpha)$. The density of $U_{n} \mid \mathbf{p}$ is

$$
f_{U_{n}}(u \mid \mathbf{p}) \propto(u+b)^{n(\mathbf{p}) \alpha-n} u^{n-1} \mathrm{e}^{-C_{\alpha, \theta}\left[(u+b)^{\alpha}-b^{\alpha}\right]}
$$


Using a Binomial expansion, the distribution of $L_{n} \mid \mathbf{p}$ is given, for all $b>0$, by

$$
f_{L_{n}}(w \mid \mathbf{p})=\frac{\sum_{k=0}^{n-1}\left(\begin{array}{c}
n-1 \\
k
\end{array}\right)(-1)^{k} w^{-k / \alpha} I\left\{w>C_{\alpha, \theta}\right\} \mathscr{G}(w \mid n(\mathbf{p}))}{\sum_{k=0}^{n-1}\left(\begin{array}{c}
n-1 \\
k
\end{array}\right)(-1)^{k} \mathbb{E}\left[G_{n(\mathbf{p})}^{-k / \alpha} I\left\{G_{n(\mathbf{p})}>C_{\alpha, \theta}\right\}\right]}
$$

The normalizing constant can be used to yield an explicit expression for the EPPF.

REMARK 18. Note that the for the range $\alpha=0$ and $b>0$, the generalized gamma process equates with the the gamma process.

\subsubsection{Generalized Inverse Gaussian}

A more challenging class is the Generalized Inverse Gaussian (GIG) class of models. First set $\theta=1$. Let $\lambda, v$ and $\delta$ be such that $0<\lambda<\infty$, while $v$ and $\delta$ are non-negative and not simultaneously 0 . As in Barndorff-Nielsen and Shephard (2001), $T$ is $\operatorname{GIG}(\lambda, \delta, v)$ if its density is of the form

$$
f_{T}(t \mid \lambda, \delta, v)=\frac{(v / \delta)^{\lambda}}{2 K_{\lambda}(\delta v)} t^{\lambda-1} \exp \left\{-\frac{1}{2}\left(\delta^{2} t^{-1}+v^{2} t\right)\right\}
$$

where $K_{\lambda}$ is a Bessel function of the third kind. When $\delta=0$ and $\lambda>0, v>0, \operatorname{GIG}(\lambda, 0, v)$ equates with the gamma distribution. When $\lambda<0, \delta>0$ and $v=0$, then $\operatorname{GIG}(\lambda, \delta, 0)$ is a reciprocal, or inverse gamma distribution. Using the parametrization, $\lambda=-a$, for $a>0$, and $b=\delta^{2} / 2$, yields the density of an inverse gamma distribution with parameters, $a, b$, with density

$$
\frac{\left(2 / \delta^{2}\right)^{\lambda}}{\Gamma(-\lambda)} t^{\lambda-1} \exp \left\{-\frac{1}{2}\left(\delta^{2} t^{-1}\right)\right\}=\frac{b^{a}}{\Gamma(a)} t^{-a-1} \exp \left\{-b t^{-1}\right\}
$$

A special case of this is when $\lambda=-1 / 2$ leading to a stable law of index $1 / 2$. The Inverse Gaussian distribution defined by setting $\lambda=-1 / 2, \delta>0$, and $v>0$ that is a $\operatorname{GIG}(-1 / 2, \delta, v)$. The hyperbolic distribution coincides with the case of $\lambda=1$. Now define,

$$
g_{|\lambda|}(x)=\frac{2}{\pi^{2}} \frac{1}{x\left[J_{|\lambda|}^{2}(\sqrt{x})+N_{|\lambda|}^{2}(\sqrt{x})\right]}
$$

where $J_{v}$ and $N_{v}$ are Bessel functions of the first and second kind respectively. The expression (41) is central to a body of work on the infinite divisibility of student t-distribution and generalized gamma convolutions. One has for $m=0,1,2 \ldots$,

$$
g_{m+1 / 2}(x)=\frac{2}{\pi^{2}} \frac{x^{(m-1) / 2}}{\prod_{i=1}^{m}\left(x+a_{j}^{2}\right)}
$$

where the $a_{1}, \ldots, a_{m}$ are the zeros of $K_{m+1 / 2}(z)$. It is known that $\mathscr{U}$ is given by

$$
\mathscr{U}(\mathrm{d} x)=I_{\left\{x \geq v^{2} / 2\right\}}\left[\delta^{2} \int_{v^{2} / 2}^{x} g_{|\lambda|}\left(2 \delta^{2} y-\delta^{2} v^{2}\right) d y+\max (0, \lambda)\right] \mathrm{d} x
$$

The simplest cases correspond to the gamma distribution, that is the Dirichlet process, and cases covered by (42). Setting $m=0, v=0$ in (42) coincides with the $\mathscr{U}_{1 / 2}$ of a stable $(1 / 2)$ law. When $v>0$, one obtains the Inverse-Gaussian distribution. Setting $\lambda=-3 / 2$ gives $m=1$, now with $v>0, \delta>0$ corresponds yields to the Thorin measure given by

$$
\mathscr{U}(\mathrm{d} y)=\frac{2}{\pi^{2}}\left(2 y-v^{2}+a_{1}^{2} \delta^{-2}\right)^{-1} I\left\{y>v^{2} / 2\right\} \mathrm{d} y
$$


The simplest case arises if one further sets $v^{2}=a_{1}^{2} \delta^{-2}$. The other cases involving (42) are a slightly more complex but certainly can be handled. For the general case, using (41) one can calculate the $\kappa_{n}$ from the moments $m_{n}$ which are obtained as ratios of Bessel functions $K_{\lambda}$. Making the substitution $u=\left(w^{2}-v^{2}\right) / 2$ for $w \geq v>0$, gives the Laplace transform and the $m_{n}$ as follows,

$$
\phi\left(\left(w^{2}-v^{2}\right) / 2\right)=\frac{v^{\lambda} K_{\lambda}(\delta w)}{w^{\lambda} K_{\lambda}(\delta v)} \quad \text { and } \quad m_{n}\left(\left(w^{2}-v^{2}\right) / 2\right)=\delta^{n} w^{-n} \frac{K_{n+\lambda}(\delta w)}{K_{\lambda}(\delta w)}
$$

The marginal density of $L_{n}=\sqrt{\left(2 U_{n}+v^{2}\right)}$ is given by,

$$
f_{L_{n}}(w)=v^{\lambda} 2^{-(n-1)}\left(w^{2}-v^{2}\right)^{n-1} w^{-(n+\lambda-1)} \delta^{n} \frac{K_{n+\lambda}(\delta w)}{K_{\lambda}(\delta v)} I\{w \geq v\}
$$

Note that one can use the further simplification for $n=0,1,2 \ldots$

$$
K_{n+1 / 2}(z)=\sqrt{\frac{\pi}{2 x}} \mathrm{e}^{-x} \sum_{k=0}^{n} \frac{(n+k) !}{2^{k}(n-k) ! k !} x^{-k}
$$

Details may be deduced from Barndorff-Nielsen and Shephard (2001).

REMARK 19. We mention again, that although the exact form of the density for $L_{n}$ or $U_{n}$ appears to be complicated, these are easily simulated using the fact that $U_{n}=\Gamma_{n} / T$. For example in the case of the inverse gamma distribution $U_{n}=\Gamma_{n} G_{a}$. The exact representation of the density is of interest for instance in possible connections and interpretations to the theory of special functions. Lijoi and Regazzini (2004) is an example of recent work exploring the interface between special functions and problems arising in Bayesian nonparametrics. See also James (2005b).

\subsubsection{First passage time distribution}

The next example, taken from Bondesson (1992, p.37), involves $\mathscr{U}$ which is a proper distribution. For simplicity set $\theta=1$. Let $1 / 2 \leq p<1$, then $T$ has a first passage time distribution if its moment generating function evaluated at $\lambda$, has the form for $b=2 \sqrt{p(1-p)} \leq 1$,

$$
\frac{1-\lambda-\sqrt{(1-\lambda)^{2}-b^{2}}}{2(1-p)}
$$

In this case, $\mathscr{U}(d y)=\frac{1}{\pi}(y-1+b)^{-1 / 2}(1+b-y)^{-1 / 2} d y$, for $1-b<y<1+b$.

\subsubsection{Some examples from the Dirichlet process mean functional}

As mentioned previously, when $\mathscr{U}$ is finite then $M_{\theta}$ has the law of a Dirichlet process mean functional. That is, taking $\mathscr{U}$ as a distribution function, the $\left(V_{i}\right)$ are iid $\mathscr{U}$. Due to the work of Cifarelli and Regazzini (1990), the law of $M_{\theta}$ is known to often have a complex density which is not commonly seen in the literature. It is of course a simple matter to then obtain an expression for the distribution of $T=G_{\theta} M_{\theta}$. Here we state two examples. First, suppose that $\theta=1$ and $1 / V_{i}$ is chosen to be a uniform distribution on $(0,1)$. Then it is known that the distribution of $M_{1}=\int_{0}^{1}(1 / y) D_{1}(d y)$ has a density given by

$$
f_{M_{1}}(v)=\frac{\mathrm{e}}{\pi} v^{-v}(1-v)^{-(1-v)} \sin (\pi v) \text { for } 0<v<1
$$

That is $\mathscr{U}(d y)=y^{-2} d y$ for $y>1$. The final example may be found in Cifarelli and Mellili (2000). Suppose that $1 / V_{i}$ is $\mathscr{B}(1 / 2,1 / 2)$, that is the Arc-sine law. Then for all $\theta>0, M_{\theta}$ is $\mathscr{B}(\theta+1 / 2, \theta+$ $1 / 2)$. 


\section{Appendix}

\subsection{Proof of Theorem 2.1, Propositions 2.1-2.3}

Proof. An intial description of the posterior distribution of $N \mid \mathbf{X}$, follows as a simple variant of Theorem 3.2 in James(2005a). First note that the result in James (2005a, Theorem 3.2) holds obviously with $h(s)$ in place of $s$. One can easily verify this by using James (2005a, Theorem 3.1). Then by using that result with $h(s, N):=h(s) / T$, it follows that the posterior distribution of $N \mid \mathbf{X}$ is equivalent to the distribution of the random measure $N_{n}^{*}=\tilde{N}+\sum_{j=1}^{n(\mathbf{p})} \delta_{J_{j, n}, Y_{j}}$, where the joint law of $\tilde{N},\left(J_{j, n}\right) \mid \mathbf{X}$ is proportional to the joint measure,

$$
\frac{1}{\left(\tilde{T}+\sum_{j=1}^{n(\mathbf{p})} h\left(J_{j, n}\right)\right)} \mathbb{P}(d \tilde{N} \mid \nu) \prod_{j=1}^{n(\mathbf{p})}\left[h\left(J_{j, n}\right)\right]^{e_{j}} \rho\left(d J_{j, n} \mid Y_{j}\right)
$$

Note that $\tilde{N}, \tilde{T}$, corresponds to $N_{n(\mathbf{p})}, T_{n(\mathbf{p})}$ and $T=\tilde{T}+\sum_{j=1}^{n(\mathbf{p})} h\left(J_{j, n}\right)$. Additionally $\mathbb{P}(d \tilde{N} \mid \nu)$ is a Poisson law with intensity $\nu$, which importantly is the same as the prior law of $N$. That is, under $\mathbb{P}(d \tilde{N} \mid \nu), \mathbb{P}(\tilde{T} \in d t)=f_{T}(t) d t$. Now an application of the gamma identity (3) yields a posterior distribution of $\tilde{N},\left(J_{j, n}\right), U_{n} \mid \mathbf{X}$ proportional to,

$$
u^{n-1} \mathrm{e}^{-u \tilde{T}} \mathbb{P}(d \tilde{N} \mid \nu) \prod_{j=1}^{n(\mathbf{p})}\left[h\left(J_{j, n}\right)\right]^{e_{j}} \mathrm{e}^{-u h\left(J_{j, n}\right)} \rho\left(d J_{j, n} \mid Y_{j}\right)
$$

The result then follows by applications of Bayes rule to (45). In particular notice that from Proposition 2.1. of James (2005a),

$$
\mathrm{e}^{-u T} \mathrm{e}^{\psi(u)} \mathbb{P}(d N \mid \nu)=\mathbb{P}\left(d N \mid \nu_{u}\right)
$$

The description of $\mathscr{M}$ appearing in Proposition 2.3 is an immediate consequence of Theorem 3.2 of James (2005a) combined with the gamma identity. $\square$

\subsection{The prediction rule}

From Propositions 2.2 and 2.3 one can derive the Bayesian prediction rule, i.e.

$$
\begin{aligned}
\mathbb{P}\left(X_{n+1} \in d x_{n+1} \mid \mathbf{X}\right) & =\frac{\mathscr{M}\left(d X_{1}, \ldots, d x_{n+1}\right)}{\mathscr{M}\left(d X_{1}, \ldots, d X_{n}\right)} \\
& =\int_{0}^{\infty} \mathbb{E}\left[P_{n}^{*}\left(d x_{n+1}\right) \mid u, \mathbf{X}\right] f_{U_{n}}(u \mid \mathbf{X}) \mathrm{d} u
\end{aligned}
$$

One can rewrite the predictive distribution as a linear combination of the measure $\eta$ and of a weighted empirical distribution as follows

$$
\mathbb{P}\left(X_{n+1} \in d x_{n+1} \mid \mathbf{X}\right)=\zeta^{(n)} \eta\left(d x_{n+1}\right)+\frac{1}{n} \sum_{j=1}^{n(\mathbf{p})} \zeta_{j}^{(n)} \delta_{Y_{j}}\left(d x_{n+1}\right)
$$

where $\zeta^{(n)}=\frac{1}{n} \int_{0}^{+\infty} \tau_{1}\left(u \mid x_{n+1}\right) f_{U_{n}}(u \mid \mathbf{X}) \mathrm{d} u$ and, for each $j=1, \ldots, n(\mathbf{p})$,

$$
\zeta_{j}^{(n)}=\int_{0}^{+\infty} \frac{\tau_{e_{j, n}+1}\left(u \mid Y_{j}\right)}{\tau_{e_{j, n}}\left(u \mid Y_{j}\right)} f_{U_{n}}(u \mid \mathbf{X}) d u=\int_{0}^{+\infty} \mathbb{E}\left[h\left(J_{j, n}\right) \mid u, \mathbf{X}\right] f_{U_{n}}(u \mid \mathbf{X}) d u .
$$

See also James (2002) and Prünster (2002). One immediately notices that, in general, the empirical distribution $\sum_{j=1}^{n(\mathbf{p})} e_{j, n} \delta_{Y_{j}} / n$ is no longer sufficient for prediction. This is in contrast to what happens with the Dirichlet process. 


\subsection{Results for weighted Poisson laws}

Suppose that $g$ is a positive measurable function, such that, without loss of generality, $\mathbb{E}[g(N)]=$ $\int_{\mathbb{M}} g(N) \mathbb{P}(d N \mid \nu)=1$. In this section we describe what happens when $P$ is governed by a weighted Poisson law $\mathbb{P}_{g}(d N \mid \nu) \propto g(N) \mathbb{P}(d N \mid \nu)$. We also highlight the case where $g(N)=T^{-q} / \mathbb{E}\left[T^{-q}\right]$ for some $-\infty<q<\infty$.

Proposition 8.1 Suppose that $P$ is governed by the weighted Poisson law $\mathbb{P}_{g}(d N \mid \nu)$ described above. Then it follows that the law of $\left(N_{n(\mathbf{p})},\left(J_{j, n}\right), \mathbf{X}, U_{n}\right)$ is proportional to

$$
g\left(\tilde{N}+\sum_{j=1}^{n(\mathbf{p})} \delta_{s_{j}, Y_{j}}\right) \mathbb{P}\left(d \tilde{N} \mid \nu_{u}\right)\left[\prod_{j=1}^{n(\mathbf{p})} \mathbb{P}\left(J_{j, n} \in d s_{j} \mid u\right)\right] \mathscr{M}(d \mathbf{X} \mid u) f_{U_{n}}(u)
$$

where the $N_{n(\mathbf{p})}:=\tilde{N}$, and otherwise the laws above correspond to those given in Theorems 2.1 and 3.1. An application of Bayes rule yields the relevant marginal and posterior distributions.

We now describe an important special case of Proposition 8.1.

Proposition 8.2 Suppose that $w_{q}=\mathbb{E}\left[T^{-q}\right]<\infty$ for some $-\infty<q<\infty$. Furthermore assume that $n+q \geq 0$, then the NRMP defined by the weigthed Poisson measure $\propto T^{-q} P(d N \mid \nu)$, has the following properties.

(i) The law of $\left(N_{n(\mathbf{p})},\left(J_{j, n}\right), \mathbf{X}, U_{n}=u\right)$ is

$$
\mathbb{P}\left(d \tilde{N} \mid \nu_{u}\right)\left[\prod_{j=1}^{n(\mathbf{p})} \mathbb{P}\left(J_{j, n} \in d s_{j} \mid u\right)\right] \mathscr{M}(d \mathbf{X} \mid u) f_{U_{n, q}}(u)
$$

where $f_{U_{n, q}}(u)=u^{q}[\Gamma(n) / \Gamma(n+q)] w_{q}^{-1} f_{U_{n}}(u)$.

(ii) The density $f_{U_{n, q}}$ corresponds to a random variable $U_{n, q} \stackrel{d}{=} \Gamma_{n+q} / T$, where $\Gamma_{n+q}$ is a gamma random variable independent of $N$, and $T$ now has marginal density $t^{-q} w_{q}^{-1} f_{T}(t)$.

(iii) The posterior distributions of $N$, and hence $P$ and $\mu$, given $U_{n, q}, \mathbf{X}$ is the same as in Theorem 2.1. The marginal distributions of $\mathbf{X}$ and $\mathbf{p}$ are given by $\int_{0}^{\infty} \mathscr{M}(d \mathbf{X} \mid u) f_{U_{n, q}}(u) d u$.

ProOF. The result follows by applying the gamma identity to $T^{-(n+q)}$.

Acknowledgements We would like to thank Lennart Bondesson for a helpful conversation and references related to the real inversion formula.

\section{REFERENCES}

Aldous, D, and Pitman, J. (2002). Two recursive decompositions of Brownian bridge. To appear in Séminaire de Probabilités XXXIX (2002). Available at arxiv.math.PR/0402399

Arratia, R.A., Barbour, A.D. and TAvaré, S. (2003). Logarithmic combinatorial structures: a probabilistic approach. EMS Monographs in Mathematics. European Mathematical Society (EMS), Zurich.

Arratia, R.A., Barbour, A. D., and Tavaré, S. (1999). The Poisson-Dirichlet distribution and the scale-invariant Poisson process Combin. Probab. Comput. 8, 407-416.

Barndorff-Nielsen, O.E. and Shephard, N. (2001). Non-Gaussian Ornstein-Uhlenbeck-based models and some of their uses in financial economics. J. R. Stat. Soc. Ser. B Stat. Methodol., 63, 167-241.

Bondesson, L. (1979). A general result on infinite divisibility Ann. Probab. 7 965-979.

Bondesson, L. (1992). Generalized gamma convolutions and related classes of distributions and densities. Lecture Notes in Statistics, 76. Springer-Verlag, New York. 
Cifarelli, D.M., and Melilli, E. (2000). Some new results for Dirichlet priors. Ann. Statist. 28, 1390-1413.

Cifarelli, D. M., and Regazzini, E. (1990). Distribution functions of means of a Dirichlet process. Ann. Statist. 18 (1990), 429-442.

Derrida, B. (1981). Random-energy models: an exactly solvable model of disordered systems. Phys. Rev. B, 24, $2613-2626$.

Doksum, K. (1974). Tailfree and neutral random probabilities and their posterior distributions. Ann. Probab., 2, 183-201.

Donnelly, P. and Grimmett, G. (1993). On the asymptotic distribution of large prime factors. J. London Math. Soc., 47, 395-404.

Engen, S. (1978). Stochastic abundance models. Chapman \& Hall, London.

Escobar, M.D. (1994). Estimating normal means with the Dirichlet process prior J. Amer. Statist. Assoc. 89 268-277.

Escobar, M.D. and West, M. (1995). Bayesian density estimation and inference using mixtures. J. Amer. Statist. Assoc. 90, 577-588.

Ewens, W. J. (1972). The sampling theory of selectively neutral alleles. Theor. Popul. Biol. 3 87-112.

Ewens, W.J. and TAvaré, S. (1995). The Ewens sampling formula. In Multivariate discrete distributions (Johnson, N.S., Kotz, S. and Balakrishnan, N., eds.). Wiley, New York.

Feller, W. (1971). An introduction to probability theory and its applications. Vol. II. Second edition John Wiley and Sons, Inc., New York-London-Sydney

Ferguson, T.S. (1973). A Bayesian analysis of some nonparametric problems. Ann. Statist. 1, 209-230.

Freedman, D.A. (1963). On the asymptotic behavior of Bayes' estimates in the discrete case. Ann. Math. Statist., 34, 1386-1403.

Grote, M. and Speed, T.P. (2002). Approximate Ewens formulae for symmetric over-dominance selection. Ann. Appl. Prob., 12, 637-663.

HJorT, N.L. (1990). Nonparametric Bayes estimators based on beta processes in models for life history data. Ann. Statist., 18, 1259-1294.

HJorT, N.L. (2003). Topics in non-parametric Bayesian statistics. In Green, P.J. et al., editors, Highly Structured Stochastic Systems. Oxford Statistical Science Series, 27, 455-478. Oxford University Press, Oxford.

Ishwaran, H. and James, L. F. (2001). Gibbs sampling methods for stick-breaking priors. J. Amer. Stat. Assoc., 96, 161-173.

Ishwaran, H. and James, L.F. (2003a). Generalized weighted Chinese restaurant processes for species sampling mixture models. Statist. Sinica, 13, 1211-1235.

Ishwaran, H. and James, L. F. (2003b). Some further developments for stick-breaking priors: finite and infinite clustering and classification Sankhyā 65 577-592.

James, L.F. (2002). Poisson Process Partition Calculus with applications to exchangeable models and Bayesian Nonparametrics. Manuscript.

Available at arxiv.math.PR/0205093

JAmes, L.F. (2005a). Poisson process partition calculus with applications to Bayesian Lévy moving averages and shot-noise processes. Ann. Statist., to appear.

Available at http://ihome.ust.hk/ lancelot/

JAmES, L.F. (2005b). Functionals of Dirichlet processes, the Cifarelli-Regazzini identity and Beta-Gamma processes. Ann. Statist., to appear.

James, L.F. (2005c). Poisson calculus for spatial neutral to the right processes. Ann. Statist., to appear.

Janson, S. (2001). Asymptotic distribution for the cost of linear probing hashing. Random Structures Algortihms, 19, 438-471.

Kent, J.T. and Tyler, D.E.(2001). Regularity and uniqueness for constrained $M$-estimates and redescending $M$ estimates. Ann. Statist. $29252-265$.

Kingman, J.F.C. (1975). Random discrete distributions. J. Roy. Statist. Soc., Series B, 37, 1-22.

KIM, Y. (1999). Nonparametric Bayesian estimators for counting processes. Ann. Statist. 27 562-588.

Kolchin, V. F.(1986) Random mappings. Translated from the Russian. With a foreword by S. R. S. Varadhan. Translation Series in Mathematics and Engineering. Optimization Software, Inc., Publications Division, New York. Lavine, M. (1992). Some aspects of Pólya tree distributions for statistical modelling. Ann. Statist. 20, 1222-1235. Lijoi, A., Mena, R.H. and Prünster, I. (2005). Hierarchical mixture modelling with normalized inverse Gaussian priors. J. Amer. Stat. Assoc., to appear.

Lijoi, A. and Regazzini, E. (2004). Means of a Dirichlet process and multiple hypergeometric functions. Ann. Probab. 32 1469-1495.

LiU, J.S. (1996). Nonparametric hierarchichal Bayes via sequential imputations. Ann. Statist. 24, 911-930.

Lo, A.Y. (1984). On a class of Bayesian nonparametric estimates: I Density estimates. Ann. Statist. 12, 351-357.

Lo, A.Y., Brunner, L.J. And Chan, A.T. (1996). Weighted Chinese restaurant processes and Bayesian mixture model. Research Report Hong Kong University of Science and Technology.

Available at http://www.erin.utoronto.ca/ jbrunner/papers/wcr96.pdf .

Mauldin, R.D., Sudderth, W.D. and Williams, S.C. (1992). Pólya trees and random distributions. Ann. Statist. 20, 1203-1221. 
McCloskey, J.W. (1965). A model for the distribution of individuals by species in an environment. Ph.D. thesis, Michigan State University.

Nieto-Barajas, L.E., Prünster, I. and Walker, S.G. (2004). Normalized random measures driven by increasing additive processes. Ann. Statist. 32, 2343-2360.

Perman, M., Pitman, J., and Yor, M. (1992). Size-biased sampling of Poisson point processes and excursions. Probab. Theory Related Fields 92, 21-39.

Pitman, J. (1995). Exchangeable and partially exchangeable random partitions. Probab. Theory Related Fields 102, 145-158.

Pitman, J. (1996). Some developments of the Blackwell-MacQueen urn scheme. In Statistics, Probability and Game Theory. Papers in honor of David Blackwell (Eds. Ferguson, T.S., et al.). Lecture Notes, Monograph Series, 30, 245-267. Institute of Mathematical Statistics, Hayward.

Pitman, J. (1997). Partition structures derived from Brownian motion and stable subordinators. Bernoulli 3, 79-96. Pitman, J. (2002). Combinatorial stochastic processes. Lecture notes for St. Flour Summer School.

Pitman, J. (2003). Poisson-Kingman partitions. In Goldstein, D.R., editor, Science and Statistics: A Festschrift for Terry Speed. Lecture Notes, Monograph Series, 40, 1-35. Institute of Mathematical Statistics, Hayward.

Pitman, J. and Yor, M. (1997). The two-parameter Poisson-Dirichlet distribution derived from a stable subordinator. Ann. Prob. 25, 855-900.

PRÜNSTER, I. (2002). Random probability measures derived from increasing additive processes and their application to Bayesian statistics. Ph.d dissertation, University of Pavia.

Regazzini, E., Lijoi, A. and Prünster, I. (2003). Distributional results for means of random measures with independent increments. Ann. Statist. 31, 560-585.

Ruelle, D. (1987). A mathematical reformulation of Derrida's REM and GREM. Comm. Math. Phys., 108, 225-239.

Steutel, F.W. and van Harn, K. (2004). Infinite divisibility of probability distributions on the real line. Monographs and Textbooks in Pure and Applied Mathematics, 259. Marcel Dekker, New York.

Thorin, O. (1977). On the infinite divisibility of the lognormal distribution. cand. Actuar. J. 3, 121-148.

Thorin, O. (1978) An extension of the notion of a generalized $\Gamma$-convolution. Scand. Actuar. J. 3, 141-149.

LANCELOT F. JAMES

Department of Information and Systems Management

Hong Kong University of Science and Technology

Clear Water Bay, Kowloon, Hong Kong

E-MAIL: lancelot@ust.hk

Antonio Lijoi, Igor Prünster

Dipartimento di Economia Politica e Metodi Quantitativi

Università Degli Studi di PAVIA

Via San Felice 7, 27100 PaVia

E-MAIL: lijoi@unipv.it, igor.pruenster@unipv.it 